\title{
Small GTPase ARF6 controls VEGFR2 trafficking and signaling in diabetic retinopathy
}

\author{
Weiquan Zhu, ${ }^{1,2,3}$ Dallas S. Shi, ${ }^{1,4}$ Jacob M. Winter, ${ }^{1}$ Bianca E. Rich, ${ }^{1}$ Zongzhong Tong, ${ }^{5,6}$ Lise K. Sorensen, ${ }^{1}$ Helong Zhao, ${ }^{1}$ \\ Yi Huang, ${ }^{6}$ Zhengfu Tai, ${ }^{6}$ Tara M. Mleynek, ${ }^{1}$ Jae Hyuk Yoo, ${ }^{1}$ Christine Dunn, ${ }^{5}$ Jing Ling, ${ }^{1}$ Jake A. Bergquist, ${ }^{1}$ Jackson R. Richards, ${ }^{1,7}$ \\ Amanda Jiang, ${ }^{1}$ Lisa A. Lesniewski, ${ }^{8,9,10}$ M. Elizabeth Hartnett, ${ }^{11}$ Diane M. Ward, ${ }^{3}$ Alan L. Mueller, ${ }^{5}$ Kirill Ostanin, ${ }^{5}$ \\ Kirk R. Thomas, ${ }^{1,12}$ Shannon J. Odelberg, ${ }^{1,2,13}$ and Dean Y. Li $i^{1,2,4,6,7,14}$ \\ 'Department of Medicine, Program in Molecular Medicine, ${ }^{2}$ Department of Internal Medicine, Division of Cardiovascular Medicine, ${ }^{3}$ Department of Pathology, and ${ }^{4}$ Department of Human Genetics, University \\ of Utah, Salt Lake City, Utah, USA. ${ }^{5}$ Navigen Inc., Salt Lake City, Utah, USA. ${ }^{\circ}$ Key Laboratory for Human Disease Gene Study, Sichuan Academy of Medical Sciences and Sichuan Provincial People's Hospital, \\ Chengdu, China, China. 'Department of Oncological Sciences and ${ }^{8}$ Department of Internal Medicine, Division of Geriatrics, University of Utah, Salt Lake City, Utah, USA. ${ }^{9}$ Ceriatric Research Education and \\ Clinical Center, VA Salt Lake City Health Care System, Salt Lake City, Utah, USA. ${ }^{10}$ Department of Nutrition and Integrative Physiology, ${ }^{11}$ John A. Moran Eye Center, ${ }^{12}$ Department of Internal Medicine, Division \\ of Hematology, and ${ }^{13}$ Department of Neurobiology and Anatomy, University of Utah, Salt Lake City, Utah, USA. ${ }^{14}$ Department of Cardiology, VA Salt Lake City Health Care System, Salt Lake City, Utah, USA.
}

The devastating sequelae of diabetes mellitus include microvascular permeability, which results in retinopathy. Despite clinical and scientific advances, there remains a need for new approaches to treat retinopathy. Here, we have presented a possible treatment strategy, whereby targeting the small CTPase ARF6 alters VECFR2 trafficking and reverses signs of pathology in 4 animal models that represent features of diabetic retinopathy and in a fifth model of ocular pathological angiogenesis. Specifically, we determined that the same signaling pathway utilizes distinct GEFs to sequentially activate ARF6, and these GEFs exert distinct but complementary effects on VEGFR2 trafficking and signal transduction. ARF6 activation was independently regulated by 2 different ARF GEFs - ARNO and GEP100. Interaction between VECFR2 and ARNO activated ARF6 and stimulated VECFR2 internalization, whereas a VEGFR2 interaction with GEP100 activated ARF6 to promote VEGFR2 recycling via coreceptor binding. Intervening in either pathway inhibited VEGFR2 signal output. Finally, using a combination of in vitro, cellular, genetic, and pharmacologic techniques, we demonstrated that ARF6 is pivotal in VEGFR2 trafficking and that targeting ARF6-mediated VEGFR2 trafficking has potential as a therapeutic approach for retinal vascular diseases such as diabetic retinopathy.

\section{Introduction}

Over 25 million people in the United States have diabetes mellitus (1) and are prone to suffer from its devastating sequelae. The metabolic derangements seen in diabetes lead to vascular pathology including nephropathy and retinopathy, the latter being the leading cause of blindness in the developed world.

The VEGF signaling pathway plays an essential role in the pathogenesis of diabetic retinopathy (2). VEGF binds to the receptor VEGFR2 (an RTK) to activate multiple downstream signals including ERK, PLC $\gamma /$ MARCKS, and p38. Effects of these signaling pathways include endothelial cell proliferation, migration, and barrier permeability. Direct targeting of VEGF using antibodies (e.g., bevacizumab) has had some success in treating diabetic retinopathy, but patients often develop resistance to the treatment

Authorship note: W. Zhu, D.S. Shi, and J.M. Winter contributed equally to this work. Conflict of interest: The University of Utah has filed intellectual property rights concerning ARF6 pathways. The authors declare the following competing financial interests: The University of Utah has licensed technology to Navigen Inc., a biotechnology company owned in part by the University of Utah Research Foundation. A.L. Mueller, K. Ostanin, Z. Tong, and C. Dunn are employees of Navigen Inc., and D.Y. Li is a cofounder of and consultant to Navigen Inc.

Submitted: November 15, 2016; Accepted: September 7, 2017 Reference information: J Clin Invest. 2017;127(12):4569-4582. https://doi.org/10.1172/JCI91770. or do not respond adequately to inhibition of VEGF (3). Hence, a deeper understanding of the pathogenesis of diabetic retinopathy is needed to develop effective therapeutic strategies.

RTKs such as VEGFR2 bind their ligands at the plasma membrane, and subsequent trafficking of these transmembrane proteins to different subcellular compartments modulates signal output (4-7). Notably, the binding of VEGFR2 to its coreceptor neuropilin-1 (NRP1) leads to trafficking of activated VEGFR2/NRP1 away from RAB5-positive endosomes that contain a tyrosine phosphatase (thereby avoiding VEGFR2 dephosphorylation) and toward EEA1-positive endosomes. This NRP1-dependent mechanism sustains VEGFR2 activation and results in increased downstream activation of ERK1/2 compared with that observed in the absence of NRP1 (8). This same coreceptor promotes VEGFR2 recycling back to the plasma membrane instead of trafficking to the lysosome for degradation (9). Thus, when and where RTKs are activated and trafficked are important factors in these signaling cascades.

Trafficking of proteins from the plasma membrane and within intracellular compartments is modulated by small GTPases, including RABs and ARFs, which are members of the RAS superfamily. Interest in RABs and ARFs has grown in light of the integral role of trafficking in signaling cascades $(10,11)$. Wheeler et al. (6) recently demonstrated that activating mutations in RAB35 are found in some human tumors. These activating mutations act as 

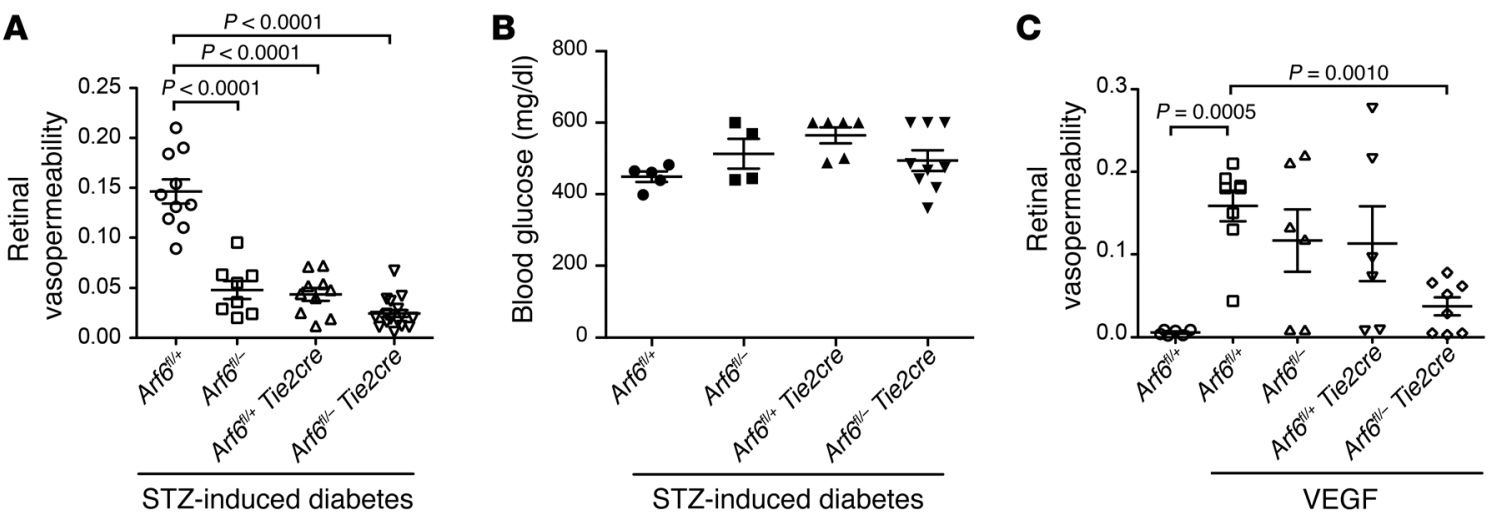

Figure 1. Homozygous and heterozygous ablation of endothelium-specific Arf6 protects against permeability in a mouse model of diabetic retinopathy. (A) STZ-induced diabetic retinal permeability in endothelium-driven Arf6-KO mice compared with that observed in littermate controls. (B) Glucose levels of mice used for A. (C) VEGF-induced retinal permeability in Arf6-conditional KO mice compared with that observed in littermate controls. Error bars represent the SEM. In $\mathbf{A}(n \geq 8)$ and $\mathbf{C}(n \geq 6)$, statistical significance was determined by Welch's 1-way ANOVA and Games-Howell multiple comparisons test for unequal variances.

oncogenes that cause constitutive PI3K/AKT activation through dysregulated endomembrane trafficking.

Small GTPases are guanine nucleotide-binding proteins that cycle between active (GTP-bound) and inactive (GDP-bound) conformations. GEFs bind GTPases and activate them by facilitating the exchange of GDP for GTP. Many GTPases can be activated by several GEFs; for example, the human genome encodes 16 ARF GEFs for the 5 ARF GTPases (12). Thus far, these multiple GEFs have been considered to have redundant functions, be differentially expressed, or to activate the same GTPase as a result of different upstream signaling pathways. For example, the Rho GEF LARG activates RhoA (another RAS family member) in response to SRC activation, while GEF-H1 activates RhoA in response to ERK activation (13). In contrast, we present data in which the same signaling pathway utilizes distinct GEFs to sequentially activate ARF 6 and exert distinct but complementary effects on VEGFR2 trafficking and signal transduction. We show that this pathway can be targeted therapeutically in 4 different animal models of diabetic retinopathy as well as in a model for ocular pathological angiogenesis.

\section{Results}

Conditional KO of Arf6 protects against vascular permeability in 2 mouse models of diabetic retinopathy. We hypothesized that the small GTPase ARF6 may be involved in the enhanced vascular permeability seen in diabetic retinopathy, as ARF6 has been shown to mediate permeability in other disease states $(14,15)$. We recently discovered the essential role of ARF6 in uveal melanoma and found upregulated protein levels of ARF6 in human tumor samples (16). Therefore, as an initial experiment, we examined ARF6 levels in postmortem whole-eye samples from patients with diabetes. We found that ARF6 appeared to be upregulated in the eyes of patients with diabetes compared with those from nondiabetic age-matched controls, although these results were not quite statistically significant $(P=0.05)$ (Supplemental Figure 1A and Supplemental Table 1; supplemental material available online with this article; https://doi.org/10.1172/JCI91770DS1). This find- ing paralleled what we discovered in tumors taken from the eyes of patients with uveal melanoma and further suggests involvement of ARF6 in disease-driven vascular remodeling.

To assess the involvement of ARF6 in diabetic retinopathy in vivo, we used the streptozotocin (STZ) mouse model. STZ is a toxic glucose analog that selectively kills pancreatic $\beta$ cells. $\beta$ Cell death causes hypoinsulinemia, leading to hyperglycemia and a phenotype strongly resembling human type 1 diabetes mellitus and diabetic retinopathy (ref. 17 and Supplemental Figure 1B). We tested the necessity of ARF6 in STZ-induced diabetic retinopathy using conditional KO mice generated by the endothelial Cre driver Tie2. Specifically, we crossed Arf $6^{A / A}$ mice with $A r f 6^{+/}$Tie2cre mice to generate compound null offspring $\left(A r f 6^{t / /}\right.$ Tie2cre, referred to hereafter as Arf6 cKO mice) and control littermates (Supplemental Figure 1C). We found that mice carrying either a single Arf6 allele or no Arf6 alleles in endothelial cells $\left(A r f 6^{+/}, A r f 6^{t /+}\right.$ Tie $2 \mathrm{cre}$, $\operatorname{Arf} 6^{A /-}$, and $A r f 6^{H /-}$ Tie2cre) showed a marked reduction in ARF6 protein levels compared with mice with 2 functional copies of Arf6 $\left(A r f 6^{+/+}, A r f 6^{A / /+}\right.$, and $A r f 6^{6 / f+7}$ ) (Supplemental Figure 1D). Our Arf6 cKO mice showed no developmental abnormalities. In particular, their retinal vascular morphology (Supplemental Figure 1E) was normal and consistent with observations made by others (18). Basal glucose levels and urine and blood tests were also indistinguishable from those of their littermates (Supplemental Figure $1 \mathrm{~F}$ and Supplemental Tables 2 and 3). Using Evans blue dye as a marker of endothelial permeability, we found that deletion of even a single allele of Arf6 protected mice from the marked increase in retinal vascular permeability in STZ-induced diabetic retinopathy (Figure 1A). This protection from excessive permeability could not be explained by differences in blood glucose levels, because STZ treatment induced fasting hyperglycemia to a similar degree in both Arf $6 \mathrm{cKO}$ and littermate control mice (Figure 1B). Therefore, endothelial ARF6 has a specific effect on the vascular sequelae of diabetes rather than on the development of diabetes itself.

The mechanisms of diabetic retinopathy are complex, but it is clear that VEGF is a key driver of pathological angiogenesis and permeability in both type 1 and type 2 diabetes. Given the marked 
A

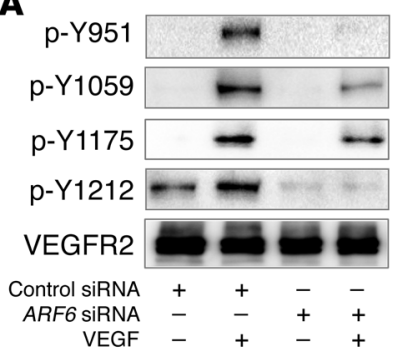

B

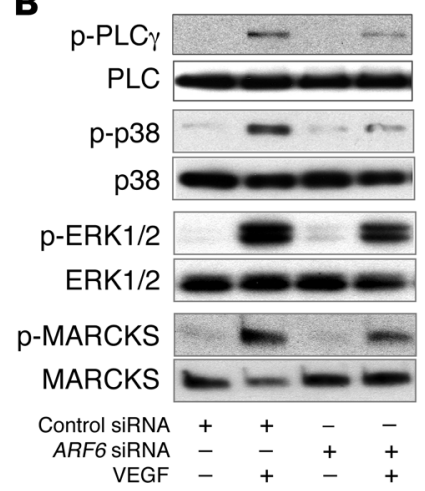

C

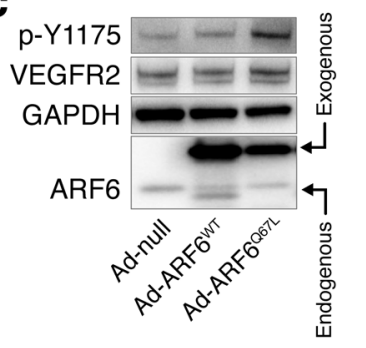

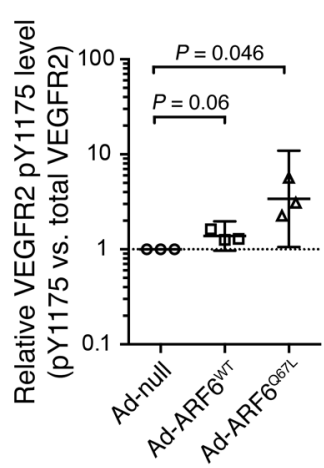

D

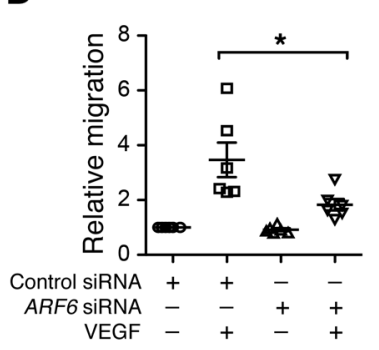

Figure 2. ARF6 is necessary for maximal VEGFR2 signaling in HRMECs. (A) ARF6 siRNA- or control siRNA-treated HRMECs were stimulated with VEGF for 5 minutes and assayed for VEGFR2 phosphorylation. (B) ARF6 siRNA- or control siRNA-treated HRMECs were stimulated with VEGF for 5 minutes and assayed for PLC $\gamma$, p38, ERK1/2, and MARCKS phosphorylation. (C) HRMECs infected with Ad-null, Ad-ARF6'WT, or Ad-ARF6 ${ }^{\text {O67L }}$ were cultured in endothelial growth media and assayed for VEGFR2 phosphorylation. (D) ARF6 siRNA-treated HRMECs were assayed for VEGF-induced migration. In $\mathbf{C}(n=3)$, geometric means and $95 \%$ Cls (error bars) of the ratios (each data point was normalized to its respective Ad-null control) were plotted on a logarithmic scale. Statistical significance was assessed using the ratio paired, 2-tailed $t$ test on non-normalized data, and the $P$ values are shown in the graph. (D) ${ }^{*} P<0.05$, by 1-way ANOVA with Tukey's multiple comparisons test $(n=6)$. All error bars represent the SEM. p, phosphorylated. effect of Arf6 deletion in the STZ model, we hypothesized that ARF6 may be necessary for VEGF signaling. To test this hypothesis, we used a VEGF-induced permeability model, in which we injected VEGF intravitreally (i.v.t.) into Arf6 cKO mice and their control littermates. As seen in the STZ model, deletion of Arf6 protected mice against VEGF-induced retinal permeability (Figure 1C). Taken together, these data support the idea that ARF6 is necessary for VEGF-induced vascular leakage in diabetic retinopathy.

ARF6 is necessary for maximal VEGFR2 signaling in human retinal microvascular endothelial cells. Our in vivo results demonstrated the necessity of ARF6 in VEGF-induced permeability, suggesting that ARF6 is required for VEGF-induced VEGFR2 activation in vivo. Indeed, we found that the eyes from $A r f 6^{\text {I/ }}$ Tie2cre mice had reduced VEGF-induced VEGFR2 phosphorylation compared with those from $\mathrm{Arff}^{A /+}$ mice (Supplemental Figure 2, A and B). Consequently, we sought to determine the underlying mechanism in vitro. We knocked down ARF6 in human retinal microvascular endothelial cells (HRMECs) using siRNA and observed that VEGFR2 activation by VEGF was greatly diminished (Figure 2A, and quantified in Supplemental Figure 2, C-G). Furthermore, downstream signaling of VEGFR2 was inhibited upon ARF6 knockdown, as indicated by reduced phosphorylation of PLC $\gamma$, p38, ERK, and MARCKS (Figure 2B, and quantified in Supplemental Figure 2, H-K). These signaling cascades represent divergent arms downstream of VEGFR2, and ARF6 knockdown inhibited each of them, positioning ARF6 at an upstream nodal point in VEGFR2 signaling. In a complementary experiment, we observed that expression of a constitutively active ARF6 mutant (ARF6 ${ }^{\text {667 }}$ ) induced VEGFR2 phosphorylation when HRMECs were cultured in endothelial growth medium, which contains only minimal VEGF (Figure 2C). These results demonstrate that ARF6 is necessary for maximal VEGFR2 phosphorylation and downstream signaling. As a functional output, we measured the migration of HRMECs in response to VEGF under different treatment conditions. ARF6 knockdown blocked VEGF-induced migration of HRMECs (Figure 2D). ARF6 knockdown also inhibited proliferation, but not apoptosis, of HRMECs in complete endothelial culture medium (Supplemental Figure 2, L and M). These results demonstrate that the effects of ARF6 on VEGFR2 have functional relevance.

Maximal VEGF signaling requires ARF6-mediated VEGFR2 trafficking in HRMECs. It has been suggested that ARF6 mediates VEGF-induced phosphorylation of caveolin-1, which is important for cell migration and proliferation in HUVECs (19). However, the role of caveolin-1 in regulating endothelial VEGF signaling is not clear and may differ among endothelial cells originating from different vascular beds (20-22). In HRMECs, VEGF stimulation did not lead to the phosphorylation of caveolin-1 (Supplemental Figure 3A), suggesting that ARF6 may affect VEGF signaling via an alternative mechanism. Previous work has shown that ARF6 functions in vesicular trafficking at the plasma membrane $(16,23)$. To determine whether the effects of ARF6 on VEGFR2 signaling were due to trafficking events, we measured endocytosis by labeling cell-surface proteins with biotin, allowing time for endocytosis and then removing biotin from proteins remaining at the cell surface. Using this assay, we found that $A R F 6$ knockdown inhibited the VEGF-induced internalization of VEGFR2 (Figure 3, A and B). This result was confirmed by immunofluorescence microscopy (Figure 3C and Supplemental Figure 3B). To determine whether this internalization is responsible for the effects of ARF6 on VEGFR2 phosphorylation, we treated HRMECs with Pitstop 2, a small-molecule inhibitor of clathrin-mediated endocytosis. We found that Pitstop 2 treatment markedly reduced ligand-induced 
A

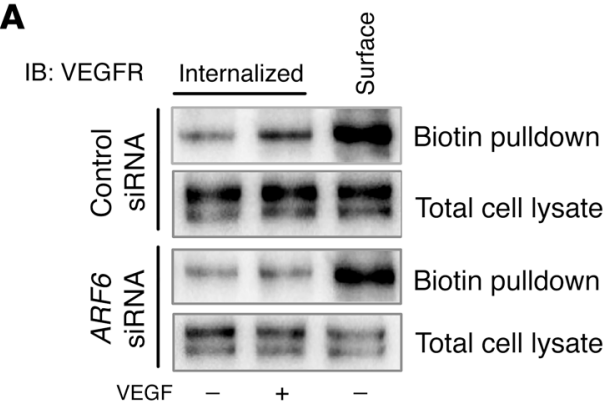

C

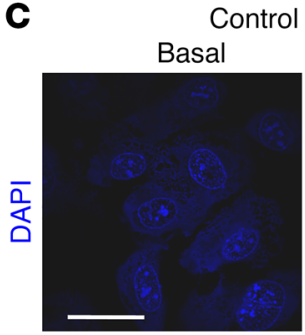

Control siRNA
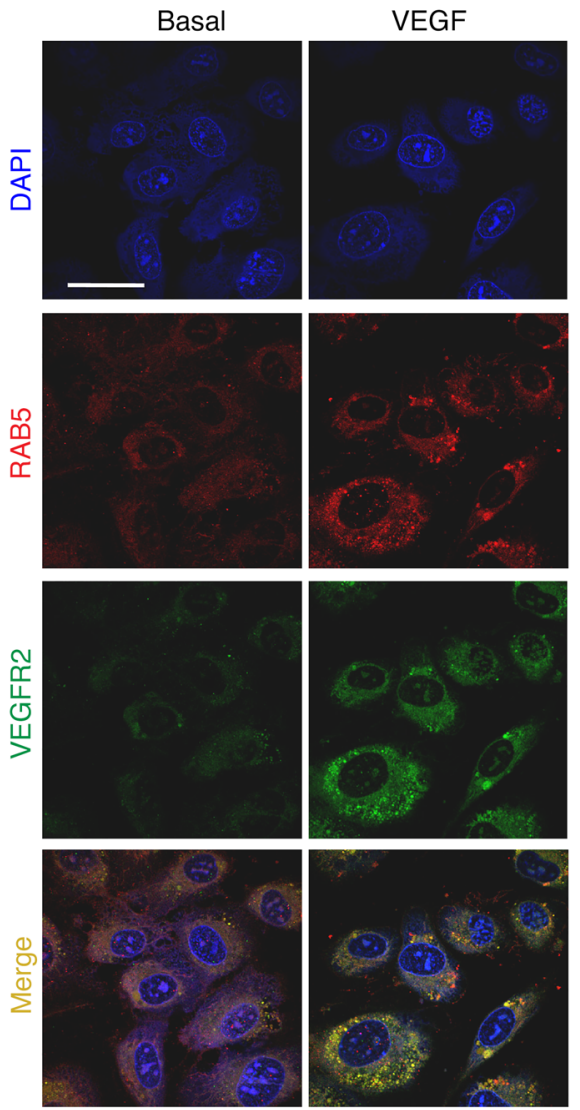

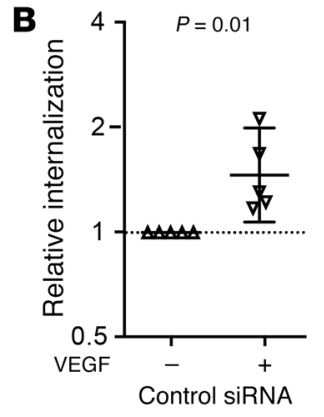

ARF6 SiRNA
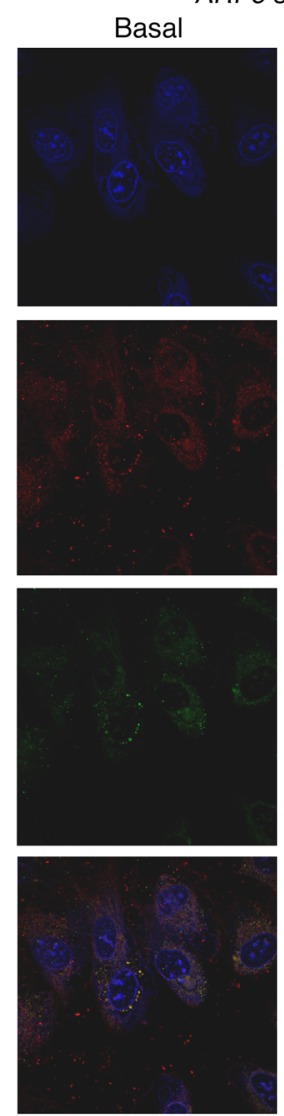
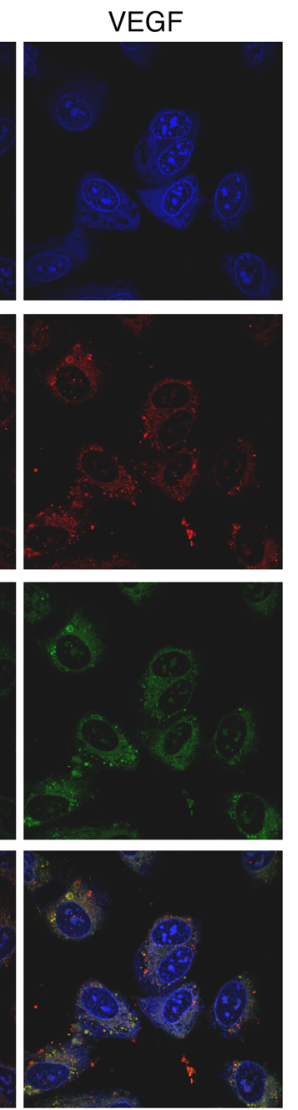

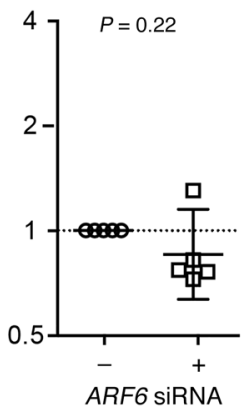

D

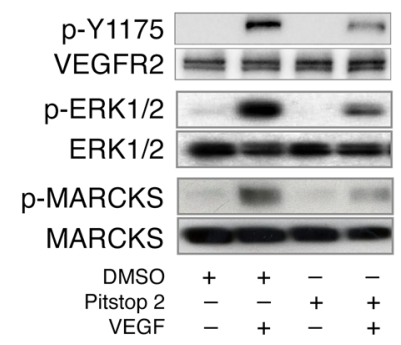

Figure 3. Maximal VECF signaling depends on ARF6-mediated receptor trafficking in HRMECs. (A and B) ARF6 siRNA- or control siRNA-treated HRMECs were labeled with biotin, stimulated with VEGF for 5 minutes, and assayed for internalized VEGFR2. IB, immunoblot. (C) ARF6 siRNA- or control siRNAtreated HRMECs were stimulated with VEGF for 5 minutes and assayed for colocalization of internalized VEGFR2 with endosome markers. Scale bar: 30 $\mu \mathrm{m}$; original magnification, $\times 1,200$. The images in C show a single optical confocal section through an internal region of the HRMECs; the Z-stacked images are shown in Supplemental Figure 3B. (D) Pitstop 2-treated HRMECs were stimulated with VEGF for 5 minutes and assayed for VEGFR2, ERK1/2, and MARCKs phosphorylation. In B $(n=5)$, geometric means and 95\% Cls (error bars) of the ratios (each data point normalized was to its respective untreated control) were calculated, and the ratios were plotted on a logarithmic scale. Statistical significance was assessed using the ratio paired, 2-tailed $t$ test on non-normalized data, and the $P$ value is shown in each graph.

VEGFR2 phosphorylation and downstream signaling, phenocopying ARF6 knockdown (Figure 3D, and quantified in Supplemental Figure 3, C-E). This result indicates that blocking clathrin-mediated endocytosis is sufficient to inhibit VEGF-induced VEGFR2 activation and signaling. Taken together, these data support a model in which ARF6 controls VEGFR2 endocytosis and consequently affects its activation and downstream signaling.

The ARF GEF ARNO is required for VEGFR2 internalization. Activation of small GTPases such as ARF6 depends on GEFs, which activate GTPases by triggering the exchange of GDP for GTP. The ARF GEF ARNO was shown to be involved in VEGFR2 signaling in an immortalized human microvascular endothelial cell line; however, ARF6 was not investigated in that study (24). We tested whether ARNO was responsible for VEGF-induced ARF6 activation in our system. Knockdown of ARNO decreased VEGF-induced ARF6 activation as measured by ARF6-GTP pulldown assays (Figure 4, A and B, and Supplemental Figure 4A). Since we observed that ARF6 activation is necessary for maximal VEGFR2 activation and signaling, 
A
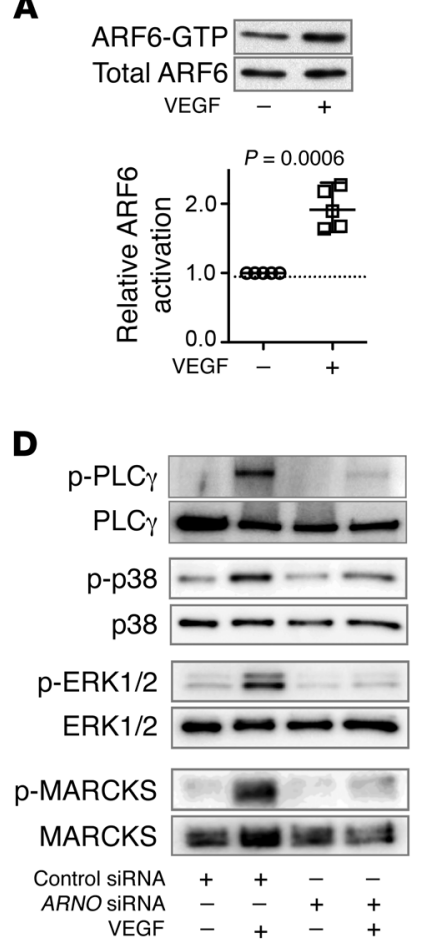

B

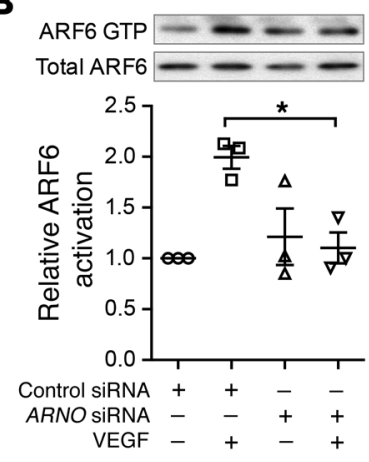

E

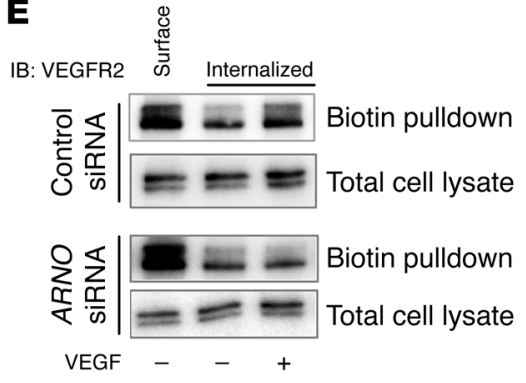

C

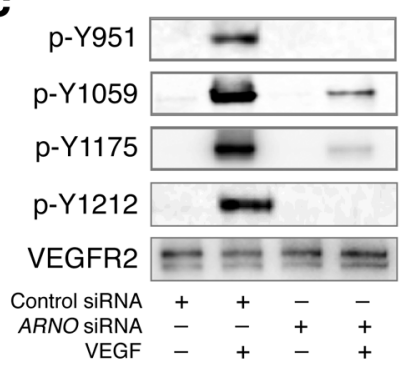

$\mathbf{F}$

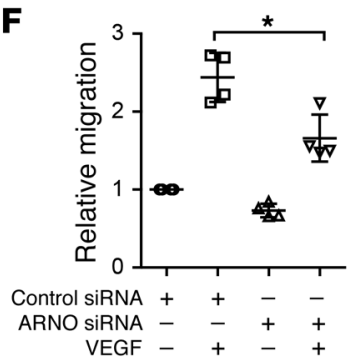

G

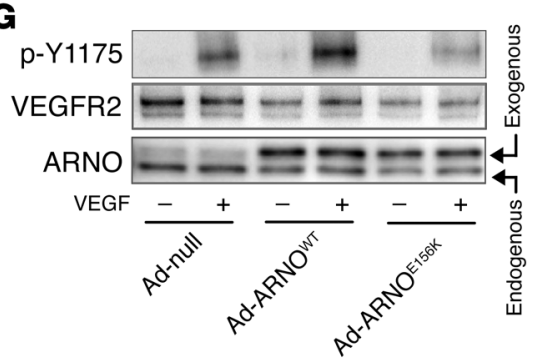

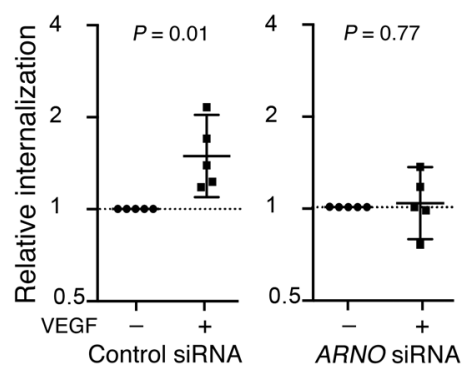

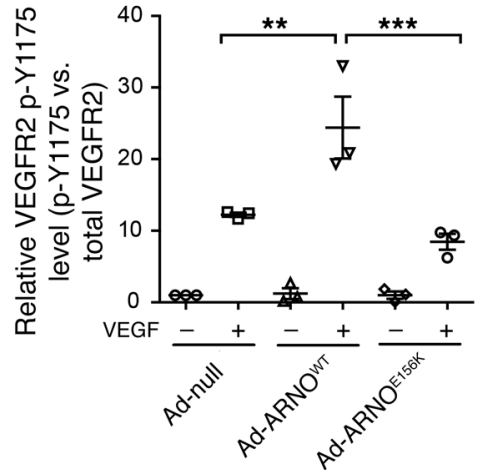

Figure 4. The ARF-GEF ARNO is required for VEGFR2 internalization. (A) VEGF-treated HRMECs were assayed for ARF6 activation using an ARF6-GTPpulldown assay. (B) ARNO siRNA-treated HRMECs were assayed for VEGF-induced ARF6 activation. (C) ARNO siRNA- or control siRNA-treated HRMECs were stimulated with VEGF for 5 minutes and assayed for VEGFR2 phosphorylation. (D) ARNO siRNA- or control siRNA-treated HRMECs were stimulated with VEGF for 5 minutes and assayed for PLC $\gamma$, p38, ERK1/2, and MARCKS phosphorylation. (E) ARNO siRNA- or control siRNA-treated HRMECs were labeled with biotin, stimulated with VEGF for 5 minutes, and assayed for internalized VEGFR2. (F) ARNO siRNA- or control siRNA-treated HRMECs were assayed for VEGF-induced cell migration. (C) HRMECs infected with Ad-null, Ad-ARNOWT, or Ad-ARNOE156K were stimulated with VEGF and assayed for VEGFR2 phosphorylation. In $\mathbf{A}(n=5)$ and $\mathbf{E}(n=5)$, geometric means and $95 \% \mathrm{Cls}$ (error bars) of the ratios (each data point was normalized to its respective untreated control) were calculated, and the ratios were plotted on a logarithmic scale. Statistical significance was assessed using the ratio paired, 2-tailed $t$ test on non-normalized data, and the $P$ value is shown in each graph. In $\mathbf{B}(n=3), \mathbf{F}(n=4)$, and $\mathbf{G}(n=3)$, a 1-way ANOVA with Tukey's multiple comparisons test was used to assess statistical significance $\left({ }^{*} P<0.05,{ }^{* *} P<0.01\right.$, and $\left.{ }^{* *} P<0.001\right)$. All error bars represent the SEM.

we reasoned that knocking down the GEF that activates ARF6 would have the same effect on VEGFR2. Indeed, we found that ARNO knockdown inhibited VEGF-induced VEGFR2 activation (Figure 4C, and quantified in Supplemental Figure 4, B-E) as well as phosphorylation of PLC $\gamma, \mathrm{p} 38$, ERK, and MARCKS (Figure 4D, and quantified in Supplemental Figure 4, F-I). Furthermore, we observed that ARNO knockdown blocked VEGFR2 internalization in our biotinylation assay (Figure $4 \mathrm{E}$ ). Last, we found that $A R N O$ knockdown inhibited VEGF-induced migration of HRMECs (Figure 4F).

To complement these loss-of-function experiments, we used adenoviral vectors to overexpress WT ARNO and a mutant of
ARNO that lacks GEF activity (ARNO ${ }^{\mathrm{E} 156 \mathrm{~K})}(25,26)$. Compared with HRMECs treated with an empty adenovirus (Ad-null), we found that Ad-ARNO ${ }^{\text {WT }}$ treatment significantly increased VEGF-induced VEGFR2 phosphorylation, while Ad-ARNO ${ }^{\mathrm{E} 156 \mathrm{~K}}$ treatment did not (Figure 4G). These data indicate that ARNO overexpression potentiates VEGF signaling and that this potentiation requires the GEF activity of ARNO, supporting our model in which ARNO activates ARF6 to facilitate VEGFR2 endocytosis and signaling.

GEP1OO is necessary for maintaining VEGFR2 protein levels. GEP100 is another GEF that has been shown to activate ARF6 (16, 27). Given that ARNO activates ARF6 in our assays, we assumed 
A ARF6-GTP Total ARF6
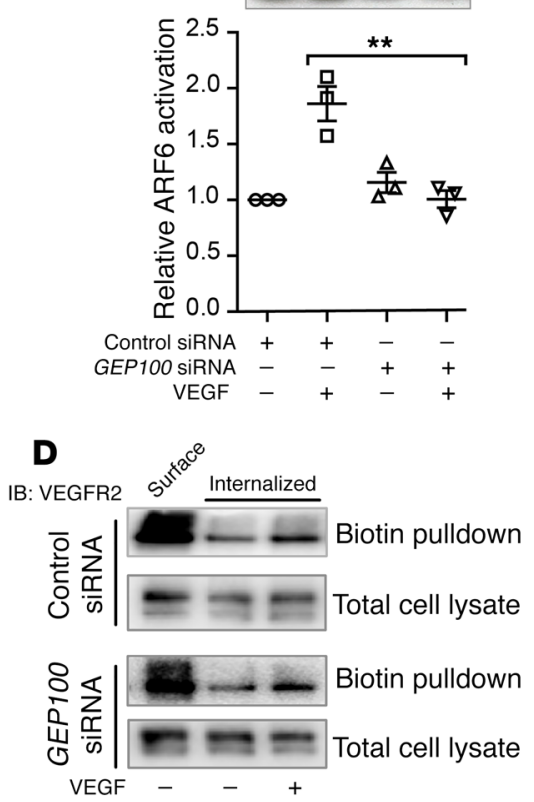

B
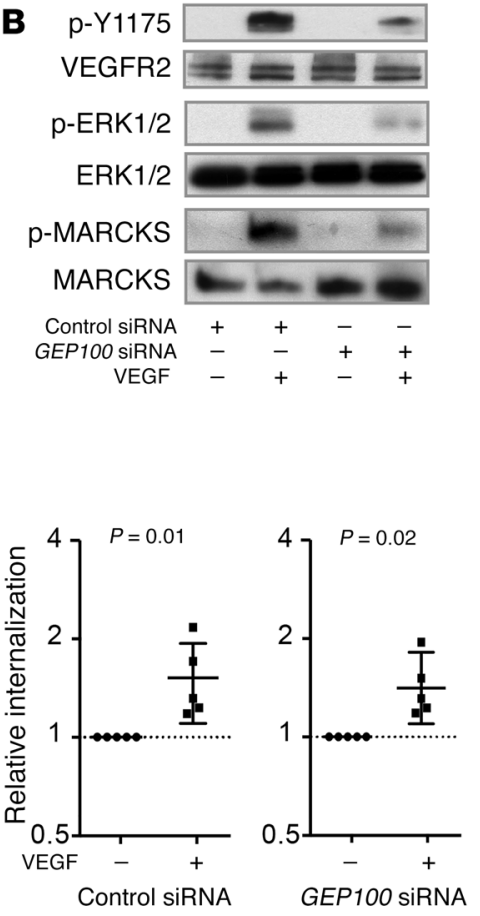

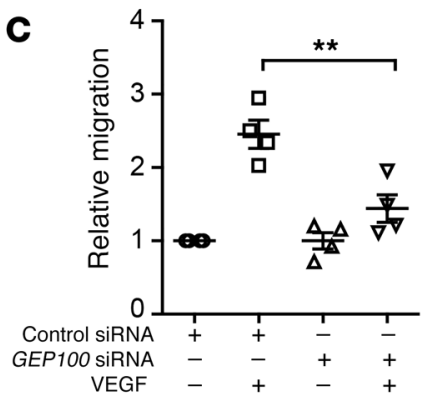

E

$\operatorname{VEGF}(\min )$

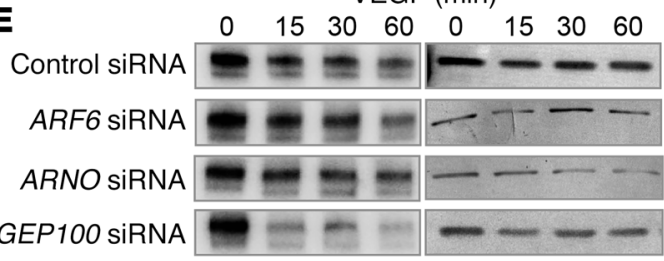

IB: VEGFR2

IB: GAPDH

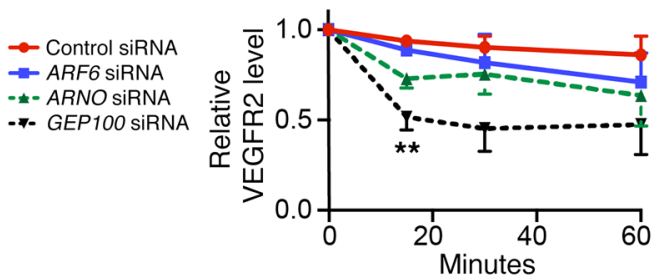

$\mathbf{F}$

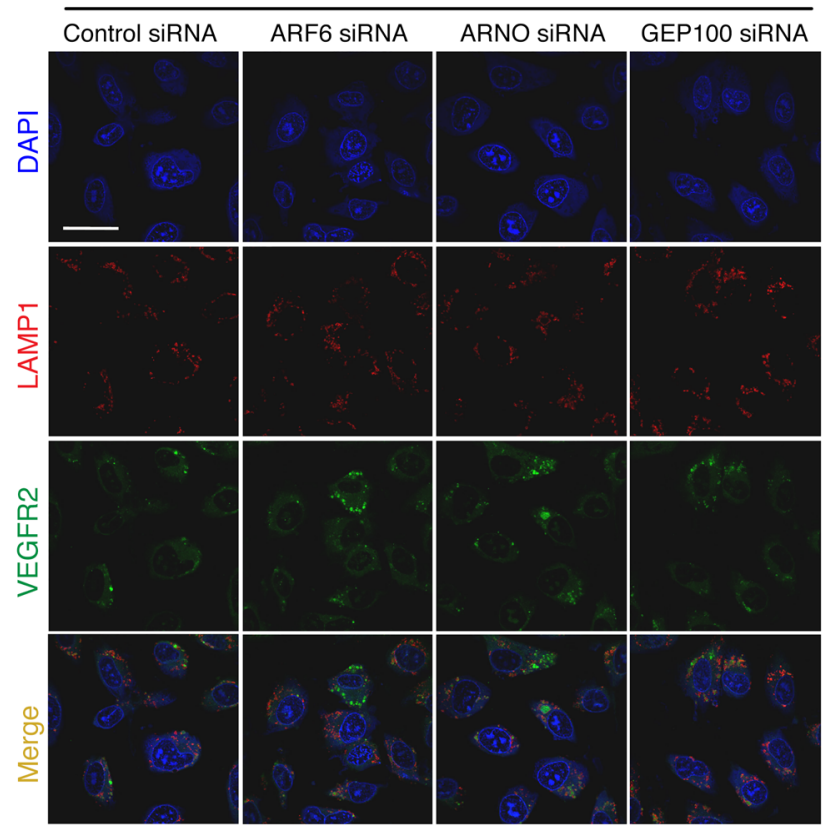

VEGF

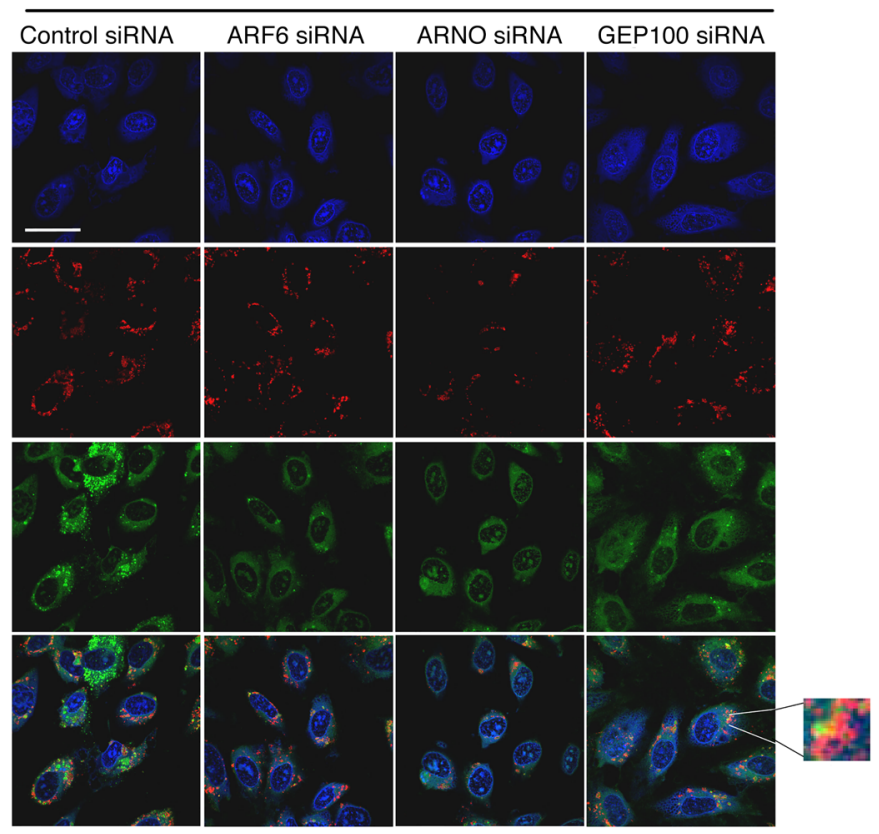

Figure 5. The ARF-GEF GEP100 is important for VEGFR2 signaling and recycling. (A) GEP100 siRNA- or control siRNA-treated HRMECs were assayed for VEGF-induced ARF6 activation. (B) GEP100 siRNA- or control siRNA-treated HRMECs were stimulated with VEGF for 5 minutes and assayed for VEGFR2, ERK1/2, and MARCKS phosphorylation. A representative blot from at least 4 independent experiments is shown. (C) GEP100 siRNA- or control siRNA-treated HRMECs were assayed for VEGF-induced cell migration. (D) GEP100 siRNA- or control siRNA-treated HRMECs were labeled with biotin, stimulated with VEGF for 5 minutes, and assayed for internalized VEGFR2. (E) ARF6 siRNA-, ARNO siRNA-, GEP100 siRNA-, or control siRNA-treated HRMECs were stimulated with VEGF for 60 minutes and assayed for VEGFR2 levels. (F) ARF6 siRNA-, ARNO siRNA-, GEP100 siRNA-, or control siRNA-treated HRMECs were stimulated with VEGF for 5 minutes and assayed for colocalization of VEGFR2 with the lysosomal marker LAMP1. Scale bars: $30 \mu \mathrm{m}$; original magnification, $\times 1,200$ and additional $\times 7$ (inset). The images in $\mathbf{F}$ show a single optical confocal section through an internal region of the HRMECs, and the Z-stacked images are shown in Supplemental Figure 4K. In D, the geometric means and 95\% Cls (error bars) of the ratios (each data point was normalized to its respective untreated control) were calculated, and the ratios were plotted on a logarithmic scale. Statistical significance was assessed using the ratio paired, 2-tailed $t$ test, and the $P$ value is shown in each graph. In $\mathbf{A}(n=3), \mathbf{C}(n=4)$, and $\mathbf{E}(n=3)$, a 1-way ANOVA with Tukey's multiple comparisons test was used to assess statistical significance for each time point $\left.{ }^{* *} P<0.01\right)$. All error bars represent the SEM. 
that GEP100 would not play a role in our HRMEC system. However, we found that knockdown of GEP1OO significantly inhibited VEGF-induced ARF6 activation (Figure 5A and Supplemental Figure 4J), VEGFR2 phosphorylation at Y1175 (Figure 5B), downstream signaling (Figure 5B), and migration (Figure 5C). These signaling data were consistent with those obtained upon knockdown of ARNO. However, further studies revealed distinct functions of GEP100 and ARNO with regard to VEGFR2 signaling. Specifically, we found that knockdown of GEP100 did not affect VEGFinduced internalization of VEGFR2 (Figure 5D), a strikingly discordant finding compared with our ARNO data. GEP10O knockdown also resulted in accelerated VEGFR2 degradation. We found that VEGFR2 protein levels decreased more rapidly after VEGF stimulation in GEP100-knockdown cells compared with cells in which $A R N O$ or ARF6 had been knocked down (Figure 5E). Furthermore, knockdown of GEP100 increased colocalization of VEGFR2 and the lysosomal marker LAMP1, as determined by immunofluorescence microscopy (Figure 5F and Supplemental Figure 4K). These data suggest that GEP100 knockdown, but not ARF6 knockdown, accelerated VEGFR2 degradation, because knockdown of ARF6 also inhibited VEGFR2 internalization, which prevented subsequent endosomal sorting to the lysosome. Together, these data indicate that GEP100 is necessary for maximal VEGFR2 activation and signaling through its role in maintaining VEGFR2 protein levels, but is dispensable for VEGFR2 internalization.

GEP10O-ARF6 promotes binding of VEGFR2 with its coreceptor NRP1, which regulates VEGFR2 intracellular trafficking and signal output. Recent seminal studies have revealed the importance of coreceptors in RTK trafficking. Specifically, it has been shown that NRP1 acts as a coreceptor for VEGFR2. When VEGFR2 binds $\mathrm{NRP} 1$, it is recycled to the plasma membrane rather than trafficked to the lysosome for degradation $(8,9,28)$. In light of these established findings, we hypothesized that ARF6 might affect VEGFR2 trafficking by influencing its interaction with NRP1. Knockdown of ARF6 prevented VEGF-induced binding of NRP1 and VEGFR2 (Figure 6A). To investigate which GEF was responsible for this function of ARF6, we knocked down GEP1OO and ARNO and found that only GEP100 knockdown affected VEGFR2NRP1 binding (Figure 6B). To test whether the ARF6-GEF activity of GEP100 is necessary for VEGFR2-NRP1 interaction, we expressed constitutively active ARF6 ${ }^{\text {Q67 }}$ in cells in which GEP100 was silenced and showed that ARF6 ${ }^{\text {Q67L }}$ rescued VEGFR2-NRP1 binding (Figure 6C). This result strongly suggests that the ARF6GEF activity of GEP100 is specifically required for the interaction of VEGFR2 and NRP1.

We next asked whether GEP100 and ARF6 affect VEGFR2NRP1 binding at the plasma membrane or after internalization. We found that GEP100 knockdown blocked VEGFR2-NRP1 binding, even in the presence of the clathrin-mediated endocytosis inhibitor Pitstop 2, suggesting that GEP100 exerts its effects on this interaction at the plasma membrane before internalization (Figure 6D). These data indicate that the action of GEP100 is to facilitate VEGFR2-NRP1 binding at the plasma membrane, but that the consequence of this VEGFR2-NRP1 binding is manifested later during subsequent intracellular trafficking and recycling. Thus, GEP100 is necessary for maximal VEGFR2 activation and maintains VEGFR2 protein levels.
To determine whether GEP100 and ARNO influence VEGFR2 trafficking at distinct locations, we examined their subcellular localization by immunofluorescence. We found that GEP100 and ARNO do not colocalize in HRMECs (Figure 6E). Upon VEGF stimulation, both ARNO and GEP100 showed punctate staining with little overlap. Some of the GEP100 protein appeared to be located near the plasma membrane, while the majority of ARNO was present in clustered cytoplasmic and perinuclear pools. This result suggests the presence of 2 spatially distinct pools of ARF-GEFs in HRMECs.

These in vitro data indicate that 2 ARF GEFs, GEP100 and ARNO, activate ARF6 to exert distinct and complementary effects on VEGFR2 trafficking and signaling. Our findings further indicate that ARNO activates ARF6 to induce VEGFR2 internalization and that GEP100 activates ARF6 to enable VEGFR2-NRP1 binding at the plasma membrane, which prevents trafficking of VEGFR2 to the lysosome for degradation after internalization. Both of these trafficking mechanisms involve ARF6 and together lead to maximal VEGFR2 signal transduction.

A small-molecule inhibitor of ARF6 reduces pathology in 2 mouse models of diabetic retinopathy and a mouse model of ocular pathological angiogenesis. Our in vitro and in vivo genetic studies provide a strong rationale for targeting ARF6 in diseases such as diabetic retinopathy that feature hyperactive VEGF signaling. Our laboratory recently developed a small-molecule inhibitor of ARF6, NAV2729 , that showed efficacy in a mouse model of uveal melanoma. We also have shown that this compound is not toxic at efficacious doses when administered systemically in several different mouse strains (16). Pharmacokinetic studies using NAV-2729 were performed in mouse eyes (Supplemental Figure 5A), and NAV-2729 was shown to be present at high levels in the eye for at least 1 week. Injection of NAV-2729 into mouse eyes decreased VEGF-induced VEGFR2 phosphorylation in vivo (Supplemental Figure 5B). Also, treatment of cultured HRMECs with NAV-2729 reduced the levels of the active, GTP-bound form of ARF6 (Figure 7A), blocked VEGF-induced VEGFR2 phosphorylation (Figure 7B), and inhibited VEGF-induced migration of HRMECs in a concentrationdependent manner (Figure 7C).

To assess the therapeutic potential of inhibiting ARF6 in the context of diabetic retinopathy, we administered this compound in the STZ mouse model. We found that NAV-2729 treatment significantly reduced vascular permeability in the retinae of diabetic mice and was more efficacious than SU4312, a small-molecule inhibitor of VEGFR2 (Figure 7D and Supplemental Figure 5, C and D).

To provide a more complete clinical representation of diabetic retinopathy in vivo, we tested the protective effects of the ARF6 small-molecule inhibitor NAV-2729 in oxygen-induced retinopathy (OIR), which has been used as a mouse model for proliferative diabetic retinopathy (29). We found that, as in mice treated with SU4312, NAV-2729 treatment significantly inhibited the extent of retinal neovascularization in OIR (Supplemental Figure 5E). To further assess the therapeutic potential of ARF6 inhibition for other angiogenic ocular diseases, we tested the effects of NAV-2729 on laser-induced choroidal neovascularization (CNV) and found that $\mathrm{CNV}$ was reduced when mice were administered this ARF6 inhibitor (Supplemental Figure 5F).

A small-molecule inhibitor of ARF6 abrogates vascular permeability and pathologic angiogenesis in a rat model of diabetic retinopa- 
A
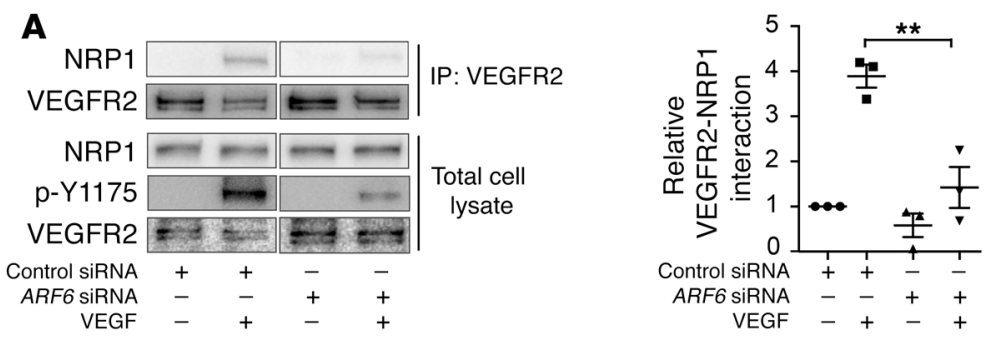

B
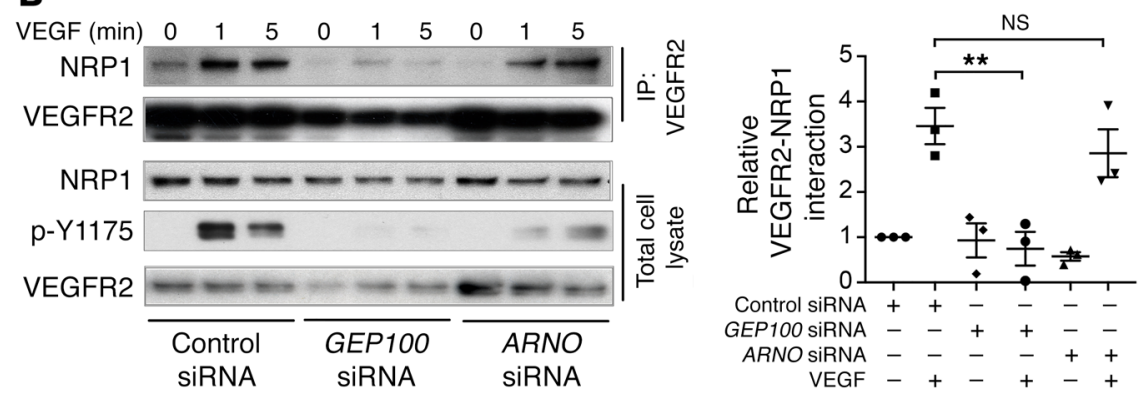

C

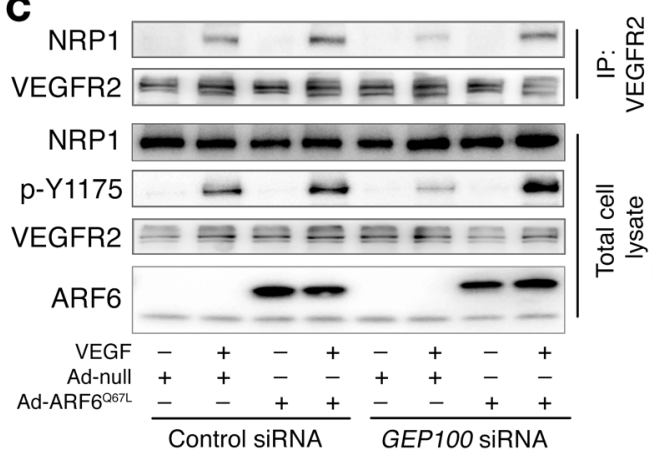

D

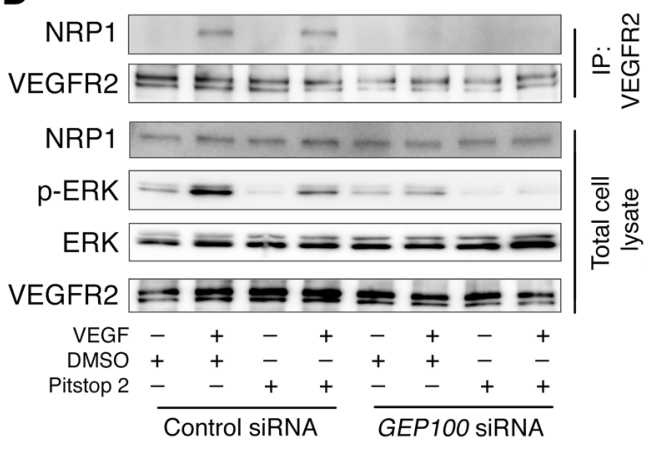

E

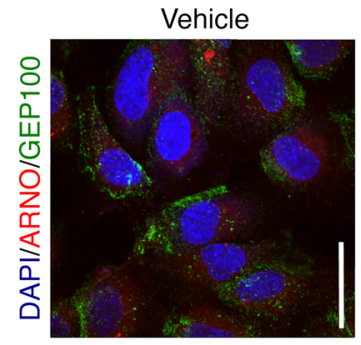

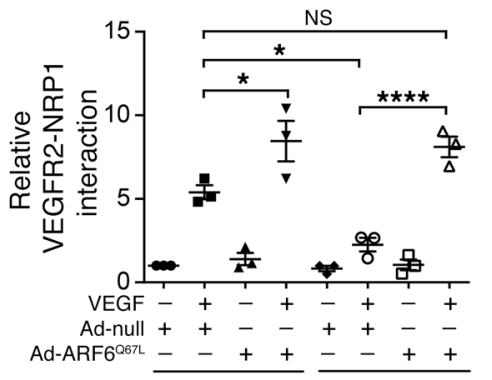

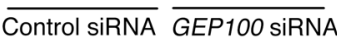

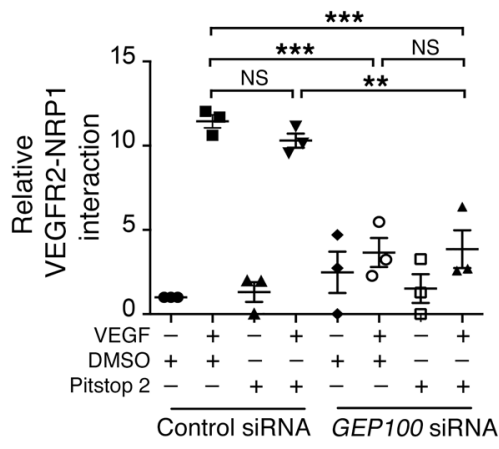

Figure 6. GEP100 activates ARF6 to promote binding of VEGFR2 and NRP1, which regulates VEGFR2 intracellular trafficking and signal output. (A) ARF6 siRNA- or control siRNA-treated HRMECs were stimulated with VEGF for 5 minutes, VEGFR2 was immunoprecipitated, and the precipitate was immunoblotted using antibodies against NRP1. Groupings of images are different parts of the same gel. (B) ARNO siRNA-, GEP100 siRNA-, or control siRNA-treated HRMECs were stimulated with VEGF for 5 minutes and assayed for VEGFR2-NRP1 coimmunoprecipitation as described for A. (C) GEP100 siRNA- or control siRNA-treated HRMECs were infected with AdARF6Q67L for 36 hours and then stimulated with VEGF for 5 minutes and assayed for VEGFR2-NRP1 coimmunoprecipitation as described for A. (D) Pitstop 2-treated and/or GEP100 siRNA- or control siRNA-transfected HRMECs were stimulated with VEGF for 5 minutes and assayed for VEGFR2/NRP1 coimmunoprecipitation as described for A. (E) HRMECs were stimulated with VEGF for 5 minutes and assayed by immunofluorescence to determine the intracellular location of ARNO and CEP100. Scale bars: $30 \mu \mathrm{m}$; original magnification, $\times 1,200$. In A-D, a 1-way ANOVA with Tukey's multiple comparisons tests was used to assess statistical significance $\left({ }^{*} P<0.05,{ }^{* *} P<0.01,{ }^{* * *} P<0.001\right.$, and ${ }^{* * * *} P<0.0001$ ). All error bars represent the SEM. $n=3$ for all panels. thy. To expand upon these findings, we further tested the efficacy of NAV-2729 in a rat model of STZ-induced diabetes. In diabetic rats, small-molecule inhibition of ARF6 significantly abrogated multiple parameters of diabetic retinopathy, including neovascu- larization, pinpoint leaks, optic disc hyperfluorescence, and vascular permeability in the retinae, without changing blood glucose levels (Figure 7, E-G, and Supplemental Figure 5, G and H). Importantly, both our mouse and rat studies involved administration of 

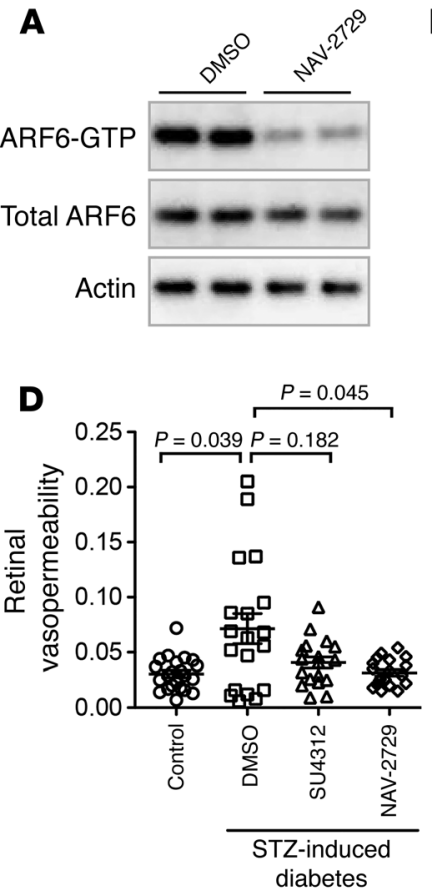

$\mathbf{F}$

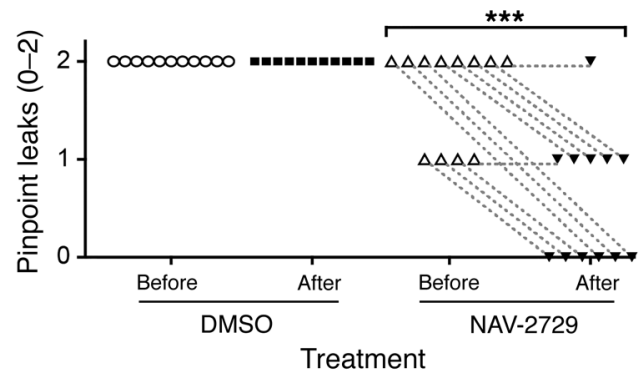

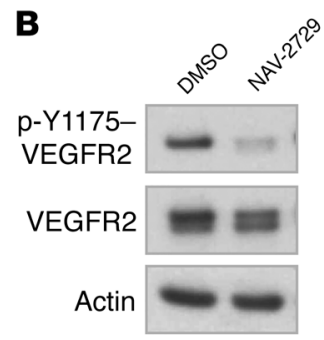
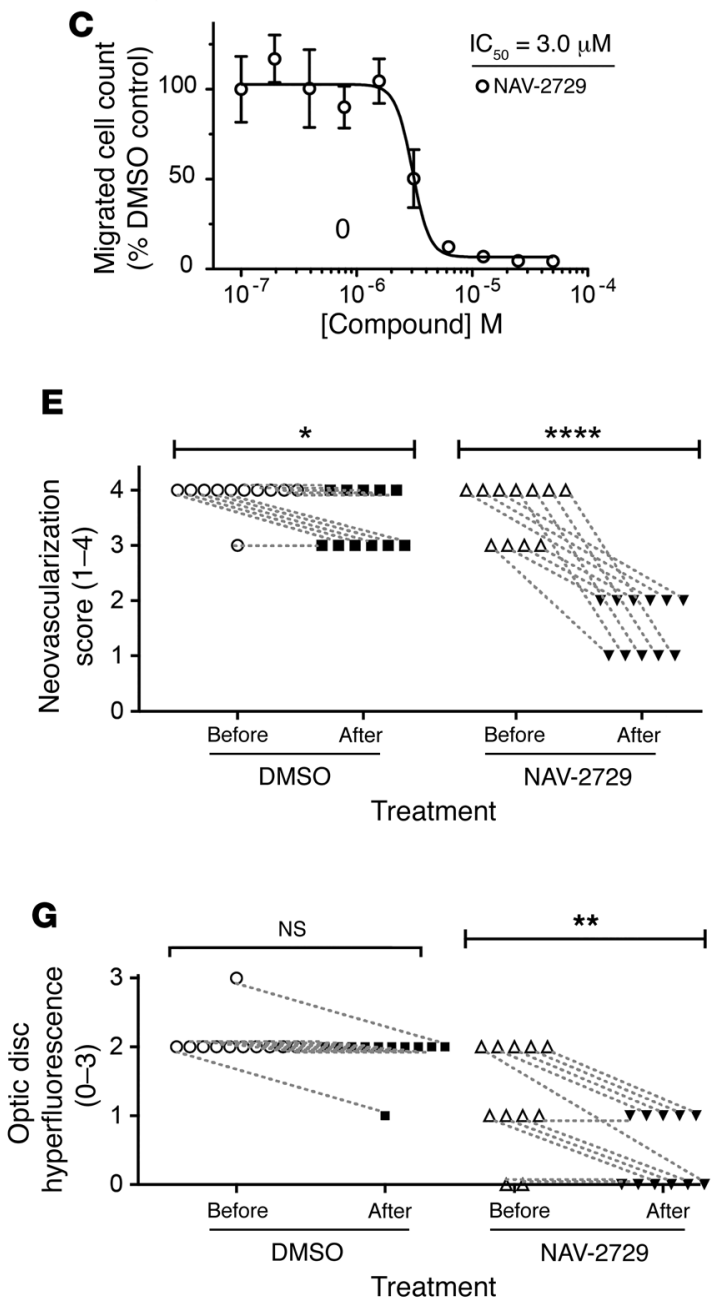

Figure 7. Pharmacological inhibition of ARF6 reverses permeability in mouse and rat models of diabetic retinopathy. (A-C) Effects of NAV-2729 on VEGF-induced ARF6-GTP levels (A), VEGFR2 phosphorylation (B), and migration (C) of HRMECs. (D) STZ-induced diabetic permeability in mice treated with DMSO, SU4312, or NAV-2729. (E) STZ-induced diabetic neovascularization in rats treated with DMSO or NAV-2729. (F) STZ-induced diabetic pinpoint leakage in rats treated with DMSO or NAV-2729. (G) STZ-induced diabetic optic disc hyperfluorescence in rats treated with DMSO or NAV-2729. Dotted lines between data points represent the same animal after different treatments. In $\mathbf{D}(n \geq 18)$, statistical significance was determined by Welch's 1-way ANOVA and Games-Howell multiple comparisons test for unequal variances. In E-G ( $n \geq 11)$, a paired, 2 -tailed $t$ test was used to assess statistical significance $\left({ }^{*} P<0.05,{ }^{* *} P<0.01,{ }^{* *} P<0.001\right.$, and $\left.{ }^{* * *} P<0.0001\right)$. All error bars represent the SEM.

NAV-2729 in the setting of established diabetes. These findings demonstrate therapeutic efficacy of ARF6 inhibition in diabetic retinopathy in 2 species.

\section{Discussion}

Our model of VEGFR2 trafficking involves 2 functionally distinct pools of ARF6, each activated by a different GEF. There is precedence for multiple GEFs working together on a single GTPase, but such data are generally interpreted as evidence of redundant, independent pathways with a common outcome (13). Here, we instead describe the action of different GEFs acting at 2 control points during the intracellular trafficking of VEGFR2. As depicted in Figure 8, activation of ARF6 by ARNO is necessary for VEGF-induced VEGFR2 internalization. Furthermore, the GEP100-dependent activation of ARF6 is necessary for VEGFR2NRP1 binding, which is required to maintain VEGFR2 protein levels and recycling of the receptor. Given these data and previ- ous reports $(8,9)$, we suggest that GEP100 functions to promote VEGFR2 recycling and prevent its lysosomal degradation by inducing the formation of VEGFR2-NRP1 complexes that are maintained throughout the signaling process. Importantly, these 2 functions of ARF 6 are distinct, but they cooperate to increase overall VEGFR2 activity. ARNO-mediated internalization increases VEGFR2 phosphorylation, while GEP100 acts to maintain this phosphorylation and total VEGFR2 protein levels. These conclusions represent a synthesis of conflicting data in the field: both ARNO and GEP100 have been independently presented as mediating VEGFR2 signaling $(24,27)$. By considering their common downstream effector ARF6, we show here that both GEFs are necessary for VEGFR2 trafficking and signaling.

In support of targeting ARF6 in diabetic retinopathy, we show that deletion of even a single allele of Arf6 in mice significantly (Figure 1A) abrogated retinal vascular leakage in the STZ model. This is not surprising, given that partial knockdown of ARF6 in 


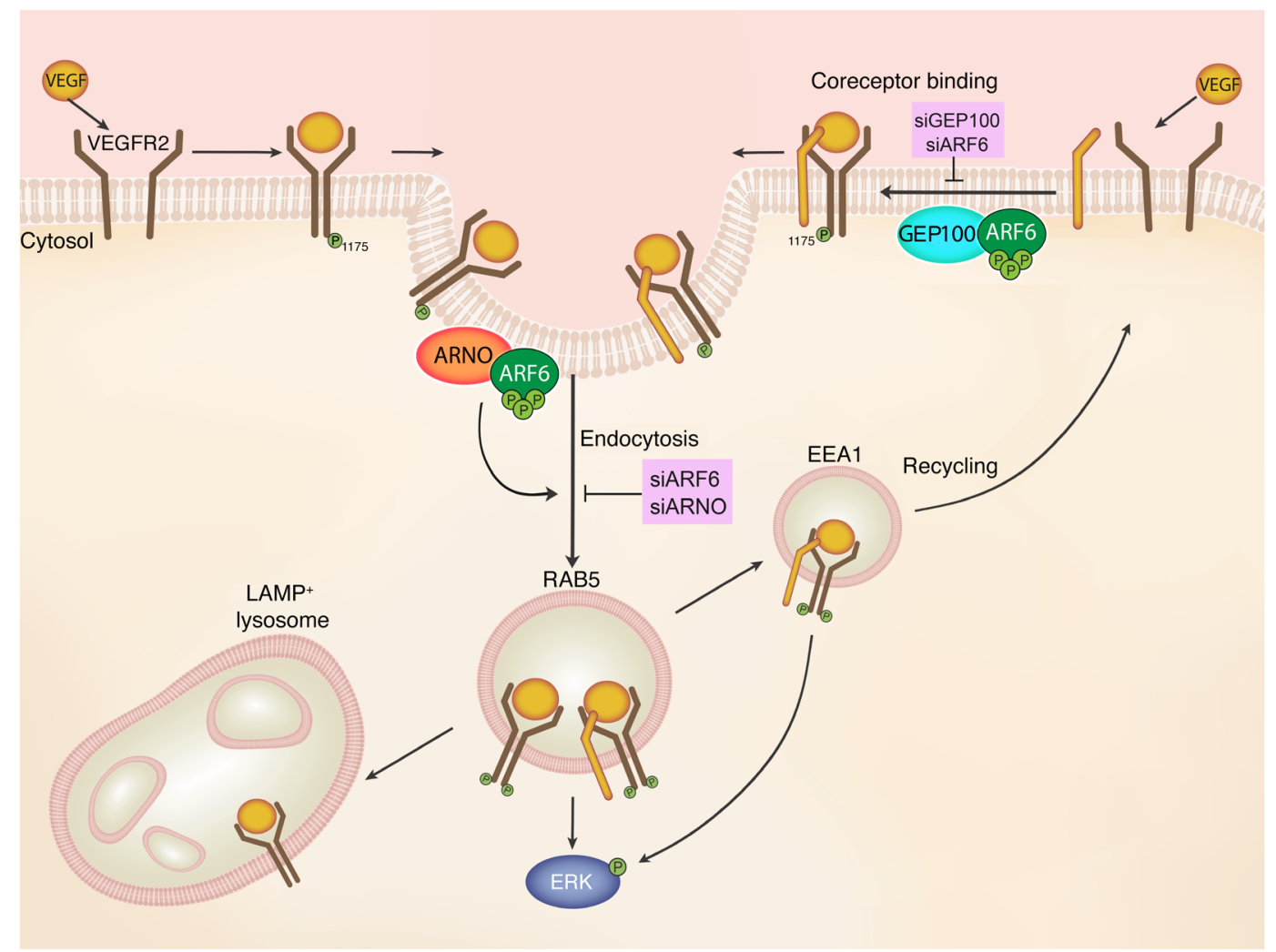

Figure 8. Schematic depicting the function of ARF6 and its GEFs in VEGFR2 trafficking and signaling. VEGF stimulation induces VEGFR2 dimerization and phosphorylation as well as ARF6 activation. The effect of ARF6 on this pathway is specified by the particular GEF that activates it. GEP100-dependent ARF6 activity promotes VEGFR2 binding to its coreceptor NRP1 at the cell surface, while ARNO-dependent ARF6 activity promotes VEGFR2 internalization. When bound to NRP1, VEGFR2 exhibits decreased lysosomal localization and degradation and increased plasma membrane recycling. Thus, ARF6 mediates VEGFR2 internalization and coreceptor binding, 2 trafficking mechanisms that are necessary for maximal VEGFR2 phosphorylation and downstream signaling. Note: phosphorylation at Y1175 has been shown to increase upon internalization of VEGFR2 (41). For simplicity, we illustrate this increase by showing only 1 of the 2 VEGFR2 chains phosphorylated at the plasma membrane. However, the true phosphorylation state of each chain at the plasma membrane has not yet been elucidated.

HRMECs significantly decreases VEGF signaling. This suggests that pharmacological inhibition of ARF6 could be a valuable therapeutic approach for treating diabetic retinopathy. A predicted reduction of approximately $50 \%$ in ARF6 protein levels is sufficient to rescue STZ-induced diabetic retinopathy, suggesting that complete inhibition of ARF6 is not necessary for therapeutic intervention. This provides us with a broad therapeutic window for delivery of the small-molecule inhibitors, which increases our chance of finding an efficacious dose that does not produce toxicity. Ablation of Arf6 using Tie2cre thus appears to inhibit pathological VEGF signaling specifically, without having detrimental effects on vascular development. In addition to our in vitro and genetic data, we show that i.v.t. injection of a small-molecule inhibitor of ARF6 in mice reduces their diabetic vascular leakage. We did find, notably, that ARF6 inhibition through genetic or pharmacological means did not alter blood glucose levels, suggesting that the phenotype presented here reflects a blockade of VEGF effects on the vasculature. These studies using STZinduced diabetes are representative of the upregulation of vascular permeability seen in early diabetic retinopathy. Given the short timeline of these studies, however, we were not able to observe differences in other pathologic signs of diabetic retinopathy such as thickening of the capillary basal lamina and neovascularization. To address these hallmarks of more advanced diabetic retinopathy, we also examined the effects of ARF6 inhibition in OIR and found that neovascularization was reduced, suggesting that ARF6 inhibition may also be an effective therapeutic approach for later stages of diabetic retinopathy.

Receptor trafficking is increasingly understood to potently regulate signal transduction. The present study changes our understanding of RTKs and GTPases, showing how 2 GEFs can use the same GTPase to exert distinct effects on a canonical RTK signaling pathway via endocytosis and recycling. ARF6 is a nodal point in the trafficking sequence of VEGFR2 and can be targeted to block multiple downstream signaling pathways in a novel manner. We show that receptor trafficking represents an effective avenue for inhibiting signal transduction in disease.

\section{Methods}

Reagents. HRMECs at passage 0 (obtained from Cell Systems) were cultured with EGM-2MV (Endothelial Cell Basal Medium-2 supplied with EGM-2 MV SingleQuots; Lonza). The following rabbit antibodies recognizing the indicated proteins were obtained from Cell Signaling Technology: p-ERK1/2, VEGFR2, VEGFR2 p-Y1175, VEGFR2 p-Y951, 
VEGFR2 p-Y1059, VEGFR2 p-Y1212, p-MARCKS, p-PLC $\gamma$, PLC $\gamma$, p-p38, p38, MARCKS, and VEGFR2 with Sepharose Bead Conjugate. Mouse anti-ERK was also obtained from Cell Signaling Technology. Mouse anti-ARF6 antibody was from MilliporeSigma or Cell Biolabs, mouse anti-VEGFR2 was from R\&D Systems, goat anti-NRP1 antibody was from Santa Cruz Biotechnology, and rabbit anti-GEP100 antibodies were from MilliporeSigma (Supplemental Tables 4 and 5). siRNAs were purchased from QIAGEN (Supplemental Table 6). ARF6-GTP-pulldown kits were from Thermo Fisher Scientific, VEGF165 was from Cell Signaling Technology, and the ChemoTx system for monitoring cell migration was from Neuro Probe. Standard molecular biology and cell culture reagents were primarily from Life Technologies (Thermo Fisher Scientific), and NAV-2729 was from Navigen Inc.

Human tissue samples. Human whole-eye samples were obtained from the Utah Lions Eye Bank. Frozen issues were homogenized on dry ice and lysed using RIPA buffer. ARF6 levels were assessed by Western blot analysis. Investigators were blinded to the patients' information and diagnosis.

Transfection and siRNAs. siRNAs were diluted in $12.5 \%$ HiPerFect Transfection Reagent (QIAGEN) in OptiMEM (Invitrogen, Thermo Fisher Scientific) and incubated for 10 to 20 minutes at room temperature. Passage 3-4 HRMECs were resuspended in EGM-2MV (Lonza) and combined with siRNAs, such that the final concentration of siRNA was $30 \mathrm{nM}$ (all targets). The cells were plated and allowed to grow overnight, and then the growth media were replaced. Three days after the initial transfection, the cells were transfected a second time using the same HiPerFect/siRNA concentrations as above.

Supplemental Table 4 lists all siRNAs used, along with their cata$\log$ numbers and sequences. We used most of these siRNAs in a previous study (14). The average knockdown was detected by Western blotting and quantified in Supplemental Figures 2 and 4 .

ARF6-GTP-pulldown assay. ARF6-GTP-pulldown assays were performed as previously described (14). Briefly, HRMECs were transfected with siRNA as described above. Cells were treated with VEGF $(20 \mathrm{ng} / \mathrm{ml})$ or vehicle for 5 minutes 72 hours after the second siRNA transfection. For assessment of the effects of small-molecule compounds on the intracellular levels of active ARF6, HRMECs were grown in EGM-2MV medium in 6-well tissue culture plates to $80 \%$ to $90 \%$ confluence and exposed to the test compound NAV-2729 (25 $\mu \mathrm{M}$ ) in basal EBM-2 medium supplemented with 1\% FBS for 3.5 hours. After treatment, media were aspirated, and cells were rinsed with chilled UltraSaline (Lonza). The dishes were frozen on dry ice. Following thawing, ARF6-pulldown lysis buffer (50 mM Tris- $\mathrm{HCl}, 100 \mathrm{mM}$ $\mathrm{NaCl}, 1 \mathrm{mM} \mathrm{MgCl}, 1 \% \mathrm{NP}-40,10 \%$ glycerol, $1 \%$ protease inhibitors [Roche] and phosphatase inhibitors [MilliporeSigma]) was added to the cells. Lysates were centrifuged, and supernatants were added to GGA3-conjugated beads (sta-40706; Cell Biolabs) and agitated at $4^{\circ} \mathrm{C}$ for 60 minutes. Beads were washed in ARF6-pulldown lysis buffer and resuspended in $2 \times$ Laemmli buffer prior to loading onto $15 \%$ SDS-PAGE gels. A fraction of the cell lysate was withheld for use as a measure of total ARF6 in each sample.

Migration assay. HRMECs were grown under standard conditions with EGM-2MV and incubated overnight in EBM-2 with $0.1 \%$ fatty acid-free BSA (Equitech-Bio Inc.). Cells were then harvested with Trypsin/EDTA (PromoCell) and resuspended at $6 \times 10^{5} \mathrm{cells} / \mathrm{ml}$. VEGF $(20 \mathrm{ng} / \mathrm{ml})$ was placed in the lower wells of a 48 -well chemotaxis chamber (Neuro Probe). A fibronectin-coated (overnight at $4^{\circ} \mathrm{C}$ ) polycarbonate membrane (10 $\mu \mathrm{m}$; Neuro Probe) was placed between the chemoattractant and the cells. HRMECs ( $50 \mu \mathrm{l}, 30,000$ cells) were added to the upper wells. After incubation at $37^{\circ} \mathrm{C}$ for 2 hours with 20 $\mathrm{ng} / \mathrm{ml}$ VEGF, cells on the top surface of the filter were removed with cotton swabs, and cells that had migrated through the filter onto the undersurface were fixed and stained using a Diff-Quik stain set (Dade Behring, Siemens). Migrated cells in 5 high-power fields were counted, and migration was expressed as the percentage of migrated cells relative to cells migrated toward EBM- 2 media containing $0.1 \%$ fatty acid-free BSA and no VEGF.

The pharmacologic effects on VEGF-induced motile activity of HRMECs were assessed using a 96-well-based ChemoTx system (Neuro Probe) (30). The basal medium EBM-2 used for this test was supplemented with $0.1 \% \mathrm{FBS}$, ascorbic acid $(1 \mu \mathrm{g} / \mathrm{ml})$, and hydrocortisone $(0.2 \mu \mathrm{g} / \mathrm{ml})$. Both polycarbonate membrane filters $(8-\mu \mathrm{m}$ pore size) and wells of the bottom (cell collector) plates were precoated with fibronectin. The $45-\mu \mathrm{l}$ aliquots of a suspension of serum-starved retinal endothelial cells in the above medium $\left(8 \times 10^{5}\right.$ cells $\left./ \mathrm{ml}\right)$ were pipetted onto the membrane filter, which was assembled on top of the collector plate. The wells of the latter were filled with the same medium supplemented with $100 \mathrm{ng} / \mathrm{ml}$ of VEGF (30 $\mu \mathrm{l} /$ well). Each test compound at a desired concentration or a DMSO control was added to the matched samples on top of the membrane and in the bottom plate. Each fully assembled ChemoTx system was incubated in a tissue culture incubator overnight, followed by aspiration of media from the top of the membrane, cell staining on both sides of the membrane using CyQUANT dye (Life Technologies, Thermo Fisher Scientific), and removal of nonmigrated cells from the top of the membrane by wiping and rinsing. Finally, the stained migrated cells were quantified fluorometrically using a Synergy 4 plate reader (BioTek), with excitation and emission wavelength settings of $485 \mathrm{~nm}$ and $528 \mathrm{~nm}$, respectively.

Immunofluorescence visualization of VEGFR2 endocytosis. VEGFR2 accumulation in vesicles was visualized according to previously described methods $(8,31)$ with the following modifications: HRMECs at passage 4 were plated onto $2 \%$ gelatin 225 bloom-coated, 8 -well chambered coverglasses at a density of $8 \times 10^{3}$ cells/well for 24 hours in complete EGM-2MV medium (Lonza). Monolayers were washed twice in EBM-2 plus $0.1 \%$ fatty acid-free BSA and starved overnight in the same media. Either $50 \mathrm{ng} / \mathrm{ml} \mathrm{VEGF}_{165}$ (Cell Signaling Technology) or HBSS vehicle was given for 5 minutes at $37^{\circ} \mathrm{C}$ and $5 \% \mathrm{CO}_{2}$, followed by 3 washes on ice for 7 minutes in ice-cold HBSS plus $\mathrm{Ca}$ and $\mathrm{Mg}, 3 \%$ fatty acid-free BSA, and $50 \mathrm{mM}$ glycine, $\mathrm{pH}$ 2.7. Cells were immediately fixed for 20 minutes at room temperature in ice-cold $10 \%$ neutral buffered formalin and washed 3 times in $1 \times$ TBS plus $\mathrm{Ca}$ and Mg. Primary antibodies against VEGFR2 (BioLegend), clathrin, and RAB5 (Cell Signaling Technology) were diluted 1:50, 1:100, and 1:50, respectively, in TBS plus $\mathrm{Ca}$ and $\mathrm{Mg}, 1 \% \mathrm{BSA}$, and $0.1 \%$ saponin and incubated overnight at $4^{\circ} \mathrm{C}$. Monolayers were rinsed 4 times in $1 \times$ TBS plus Ca and Mg. Fluorescent secondary antibodies (Alexa Fluor 488- or 594-conjugated donkey anti-mouse or anti-rabbit IgG) were diluted to $10 \mu \mathrm{g} / \mathrm{ml}$ in $1 \times$ TBS plus $\mathrm{Ca}$ and $\mathrm{Mg}, 1 \% \mathrm{BSA}$, and $0.1 \%$ saponin and applied for 1 hour at room temperature. Chambers were rinsed 3 times in $1 \times$ TBS plus $\mathrm{Ca}$ and $\mathrm{Mg}$ and mounted in $80 \%$ glycerol containing $20 \mathrm{mM}$ Tris, pH 8.0, $2 \mu \mathrm{g} / \mathrm{ml}$ DAPI, 0.5\% n-propyl gallate, $0.02 \%$ DABCO, $0.01 \% p$-phenylenediamine, and $0.02 \%$ NaZ. Z-depth images of stained cells were collected on an Olympus FV1000 confocal laser-scanning microscope, under oil immersion 
at $\times 1,200$ magnification, with seven $0.2-\mu \mathrm{m}$ slices per $Z$-stack and a minimum of 5 fields imaged per condition.

Biotinylation assay. HRMECs were transfected with ARF6, ARNO, GEP100, or control siRNA and grown to $100 \%$ confluence on $60-\mathrm{mm}$ dishes. Cells were washed twice with room-temperature PBS and incubated with $0.6 \mathrm{mM}$ primaquine (MP Biomedicals) at $37^{\circ} \mathrm{C}$ for 10 minutes to block recycling during the internalization step $(32,33)$. The cells were washed twice with ice-cold PBS and labeled with EZ-Link Sulfo-NHS-SS-Biotin (Thermo Fisher Scientific) at 0.5 $\mathrm{mg} / \mathrm{ml}$ in PBS at $4^{\circ} \mathrm{C}$ for 60 minutes. The excess biotin was removed by washing the cells with ice-cold glycine in PBS with $\mathrm{Ca}^{2+}$ and $\mathrm{Mg}^{2+}$. The cells were then incubated with $20 \mathrm{ng} / \mathrm{ml} \mathrm{VEGF}$ and 0.6 $\mathrm{mM}$ primaquine at room temperature for 5 minutes. They were then exposed twice to GSH buffer (50 mM glutathione, $75 \mathrm{mM} \mathrm{NaOH}, 75$ $\mathrm{mM} \mathrm{NaCl}, 1 \mathrm{mM}$ EDTA, 0.1\% BSA, pH 9.0) on ice for 20 minutes to remove surface biotin. GSH was quenched by washing with $5 \mathrm{mg} / \mathrm{ml}$ ice-cold iodoacetamide in PBS with $\mathrm{Ca}^{2+}$ and $\mathrm{Mg}^{2+}$. After an additional wash with ice-cold PBS, cells were lysed on ice in Pierce IP Lysis Buffer (Thermo Fisher Scientific) with a protease inhibitor cocktail. Cell lysates were centrifuged at 13,000 $\times g$ for 5 minutes, and the supernatant was incubated with High Capacity Streptavidin Agarose Resin (Thermo Fisher Scientific) at $4^{\circ} \mathrm{C}$ for 60 minutes. Beads were washed 3 times with Pierce IP Lysis Buffer. Bound proteins were released in $2 \times$ Laemmli buffer with $5 \% \beta$-mercaptoethanol at $95^{\circ} \mathrm{C}$ for 5 minutes, and internalized VEGFR2 was analyzed by immunoblotting with VEGFR2 antibody.

Immunoprecipitation. Immunoprecipitation assays were performed as previously described (34). Briefly, HRMECs were treated with $20 \mathrm{ng} / \mathrm{ml}$ VEGF for 1 or 5 minutes. Cells were then washed with ice-cold PBS and lysed with ice-cold lysis buffer (50 mM Tris- $\mathrm{HCl}$, $250 \mathrm{mM} \mathrm{NaCl}, 1 \% \mathrm{NP}-40$, and 10\% glycerol) with protease and phosphatase inhibitors (Thermo Fisher Scientific). Cell lysates were centrifuged for 15 minutes at 13,000 $\times g$ and the supernatants saved. BCA assays (Pierce, Thermo Fisher Scientific) were performed to determine protein concentrations. Lysates were precleaned with protein A/G-Sepharose (Santa Cruz Biotechnology) for 2 hours at $4^{\circ} \mathrm{C}$. Precleaned lysates were incubated with $8 \mathrm{mg}$ of the indicated antibody and protein A/G-Sepharose (Santa Cruz Biotechnology) for 1 hour at $4^{\circ} \mathrm{C}$. Beads were then washed 5 times in lysis buffer. The immunoprecipitates were assayed by Western blotting using peroxidaseconjugated AffiniPure Light Chain Specific secondary antibody (Jackson ImmunoResearch) for signal detection.

Immunofluorescence. HRMECs, at passages 3 to 5, were plated onto $10 \mu \mathrm{g} / \mathrm{ml}$ fibronectin-coated (AlphaAesar), 8-well chambered coverglasses at a density of $5 \times 10^{3} /$ well and allowed to grow for 24 hours before overnight serum starvation in EBM-2 (Lonza) plus 0.1\% BSA. Cells were treated for 5 minutes with $50 \mathrm{ng} / \mathrm{ml}$ recombinant human VEGF $_{165}$ (Cell Signaling Technology) or HBSS vehicle, followed by washing on ice in HBSS plus $\mathrm{Ca}$ and $\mathrm{Mg}$ containing $50 \mathrm{mM}$ glycine and $3 \% \mathrm{BSA}$ ( $\mathrm{pH}$ 2.7) to clear ligands. Monolayers were fixed for 20 minutes at room temperature in freshly prepared $10 \% \mathrm{v} / \mathrm{v}$ neutral buffered formalin. Formalin was cleared with 4 rinses in $1 \times$ TBS plus $\mathrm{Ca}$ and $\mathrm{Mg}$. Primary antibodies against GEP100 (IQSEC1, ab12242; Abcam) and ARNO (Cytohesin 2, ab2728; Abcam) were simultaneously diluted 1:100 each in $1 \times$ TBS plus $\mathrm{Ca}$ and $\mathrm{Mg}$ containing $1 \% \mathrm{BSA}$ and $0.1 \%$ saponin and applied to monolayers overnight at $4^{\circ} \mathrm{C}$. Unbound primary antibodies were removed with 4 rinses in $1 \times$ TBS plus $\mathrm{Ca}$ and $\mathrm{Mg}$. Sec- ondary antibodies, donkey anti-rabbit IgG, Alexa 488 conjugate, and donkey anti-mouse IgG Alexa 594 (Molecular Probes) were used at $10 \mu \mathrm{g} / \mathrm{ml}$ each in $1 \times$ TBS plus Ca and $\mathrm{Mg}$ containing $1 \%$ BSA and $0.1 \%$ saponin for 1.5 hours on ice, followed by 4 rinses in $1 \times$ TBS plus $\mathrm{Ca}$ and $\mathrm{Mg}$ and mounting in 90\% glycerol containing $5 \mu \mathrm{g} / \mathrm{ml}$ DAPI, $200 \mathrm{mM}$ Tris (pH 8.0), $0.5 \% n$-propyl gallate, $0.05 \%$ DABCO, $0.01 \%$ p-phenylenediamine, and $0.02 \%$ sodium azide.

Animals. The Arf6 gene was mutated by homologous recombination in mouse embryonic stem (ES) cells. Two alleles were generated. The conditional $(f l)$ allele contains 1 loxP site $5^{\prime}$ of the Arf6 gene at position 69,371,642 (UCSC genome browser) on chromosome 12 and a second loxP site $3^{\prime}$ of the Arf6 ORF at position 67,373,928. The null (-) allele was generated by deleting the sequences between the 2 loxP sites by Cre-mediated recombination using HPRTcre (The Jackson Laboratory). Conditional KO of Arf6 was achieved by crossing $A r f 6^{f / l}$ mice with $\mathrm{Arf6}^{+/-}$Tie2 $\mathrm{cre}$ mice (The Jackson Laboratory).

In vivo activation of VEGFR2. Twelve-week-old mice were anesthetized with ketamine- $\mathrm{HCl}(100 \mathrm{mg} / \mathrm{kg})$ and xylazine $(13 \mathrm{mg} / \mathrm{kg})$ and then administered tail vein injections of $3 \mu \mathrm{g}$ VEGF in saline. One minute later, the mice were sacrificed, and the eyes and lungs were collected and snap-frozen in liquid nitrogen. The tissue was ground and homogenized in IP lysis buffer (Pierce, Thermo Fisher Scientific), centrifuged for 15 minutes at $13,000 \times g$, and the supernatants saved. VEGFR2 was immunoprecipitated from the eye lysates as described above in the Immunoprecipitation section. Tissue lysates were mixed in $2 \times$ Laemmli buffer with $5 \% \beta$-mercaptoethanol at $95^{\circ} \mathrm{C}$ for 5 minutes, and activated VEGFR 2 was analyzed by immunoblotting with VEGFR2 antibody.

Ocular pharmacokinetics. NAV-2729 (50 ng) in $1 \mu \mathrm{l}$ of DMSO was injected into the posterior chamber of each mouse eye. Assuming that the volume of the posterior chamber of each eye is $5 \mu \mathrm{l}$ (including the $1 \mu \mathrm{l}$ injection volume), injection into both eyes will yield a NAV-2729 concentration of $100 \mathrm{ng} / 10 \mu \mathrm{l}$ for each mouse. Eyes were harvested $1,2,4$, and 7 days after injection ( $n=3-4$ mice per time point), and both eyes from 1 mouse were pooled for pharmacokinetic analysis. Eyes were weighed, diluted 10 -fold w/v in $2 \%$ nonfat milk in Dulbecco's PBS (final NAV-2729 concentration of $1,000 \mathrm{ng} / \mathrm{ml}$ at the time of injection), and homogenized with an Omni Bead Ruptor 24 homogenizer (zirconium oxide bead type, 2.8-mm bead size). Protein precipitation was induced by acetonitrile/acetone (60:40 v/v), and phospholipids were removed using PHREE 96-well plates. Concentrations of NAV-2729 in eyeball and plasma samples were determined by liquid chromatography/mass spectrometry (LC/MS) methods using an Agilent $6545 \mathrm{Q}$-TOF mass spectrometer. The lower limit of quantitation (LLOQ) was $10 \mathrm{ng} / \mathrm{ml}$.

Retinal permeability. Retinal permeability was assessed as previously described by others (35-37). In brief, 8- to 10-week-old mice were anesthetized with ketamine- $\mathrm{HCl}(100 \mathrm{mg} / \mathrm{kg})$ and xylazine $(13 \mathrm{mg} / \mathrm{kg})$ and then given intraocular injections of $1 \mu \mathrm{l}$ of 50 ng VEGF-165 (Cell Signaling Technology). An equivalent volume of VEGF in 100\% DMSO was injected into the eyes of the control animals. A half hour later, $50 \mu \mathrm{l}$ of $60 \mathrm{mg} / \mathrm{ml}$ Evans blue solution (MilliporeSigma) in UltraSaline was injected into the tail vein. After 5 hours, mice were sacrificed, the eyes were enucleated, and the retinae dissected. Dye was eluted in $0.4 \mathrm{ml}$ formamide for 18 hours at $70^{\circ} \mathrm{C}$. The extract was centrifuged at 13,000 $\times g$ for 5 minutes. Absorbance at $620 \mathrm{~nm}$ was measured. Background absorbance was measured at $740 \mathrm{~nm}$ and subtracted out. 
STZ-induced diabetic retinopathy. Diabetic retinopathy was induced as previously described by others (35). Briefly, for 5 consecutive days, 6- to 8-week-old CD1 mice (Charles River Laboratories) or conditional Arf6-KO mice were fasted for 4 hours and then injected i.p. either with $75 \mathrm{mg} / \mathrm{kg} \mathrm{STZ}$ (MilliporeSigma) in sodium citrate buffer or with sodium citrate buffer control. After 4 weeks, tail-snip blood glucose was determined using a glucometer (Aviva) to verify hyperglycemia (blood glucose concentrations $>300 \mathrm{mg} / \mathrm{dl}$ ) in the STZ-treated animals. The animals were then divided into treatment groups, such that the groups had similar distributions of body weight and blood glucose measurements. Retinal permeability was assessed as explained above.

Rat diabetic retinopathy studies were conducted in brown Norway rats by Absorption Systems, using their standard procedures. Threemonth-old male brown Norway rats (Charles River Laboratories) were injected with a single i.p. dose of $60 \mathrm{mg} / \mathrm{kg} \mathrm{STZ}$ (MilliporeSigma) in sodium citrate buffer. Blood glucose levels were measured at least once daily for the duration of the study. NAV-2729 $(5 \mu \mathrm{g} / 5 \mu \mathrm{l})$ or DMSO (5\%) was injected i.v.t. 7-8 weeks after STZ injection. A second NAV-2729 or DMSO i.v.t. injection was administered 1 week after the first i.v.t. injection. Fluorescein angiographic images were taken 3-5 days after the second i.v.t. injection. Optic disc hyperfluorescence, pinpoint leaks, and neovascularization (38) were scored later (Supplemental Table 7).

Laser-induced CNV. CNV studies were conducted by Comparative Ophthalmic Research Laboratories (University of Wisconsin, Madison, Wisconsin, USA) using their standard procedures. Two- to threemonth-old mice were anesthetized with ketamine hydrochloride (100 $\mathrm{mg} / \mathrm{kg}$ ) and their pupils dilated with $1 \%$ tropicamide (Alcon). An Iridex OcuLight GL 532-nm laser photocoagulator (Iridex) with a slitlamp delivery system was used to create 3 burns 3 disc diameters from the optic disc at 3, 6, and 9 o'clock with the following parameters: 150 $\mathrm{mW}$ power, 75-mm spot size, and 0.1-second duration. Production of a bubble at the time of laser treatment, indicating rupture of Bruch's membrane, is an important factor in obtaining CNV; therefore, only burns in which a bubble was produced were included in this study. Immediately after laser treatment and 7 days later, mice were given an i.v.t. injection of NAV-2729, SU4312, or DMSO into both eyes $(2 \mu \mathrm{l} /$ eye). Fourteen days after laser treatment, the mice were sacrificed, and choroidal flat mounts were generated and stained with ICAM-2 to visualize neovascularization by fluorescence microscopy. An investigator blinded to the treatment examined the flat mounts using a Zeiss LSM 510 confocal microscope (Carl Zeiss) and performed quantification using Image J software (NIH) to calculate the area of CNV.

OIR. P7 pups, along with nursing mothers, were placed in $75 \% \mathrm{O}_{2}$. The $\mathrm{O}_{2}$ concentration was maintained by a Pro-OX oxygen controller (Bio-Spherix). Pups were removed on P12 and given an intraocular injection of $5 \mu \mathrm{g}$ NAV-2729 or SU4312. Mice were sacrificed on P17, and their eyes were enucleated and fixed for 2 hours in $4 \%$ paraformaldehyde. Retinae were then dissected and stained overnight using Alexa Fluor 488-conjugated isolectin (1:50; Invitrogen, Thermo Fisher Scientific). Retinal flat mounts were generated and images taken using an Axiovert 200 fluorescence microscope (Carl Zeiss). Neovascularization was quantified using ImageJ software by an investigator blinded to the treatment.

Fluorescein angiography. After anesthesia, the pupils were dilated with $1 \%$ tropicamide (Bausch \& Lomb), and the cornea was kept moist using Goniovisc 2.5\% (hypromellose; MilliporeSigma). The mice were injected i.p. with $10 \%$ fluorescein sodium (Lite; Apollo Ophthalmics) at a dose of $1.5 \mathrm{ml} / \mathrm{kg}$. As is standard procedure in the field (39), several fundus images were acquired over a 5-minute period following the injection of fluorescein sodium at a rate of 4 frames per second (fps) using a Micro-III retinal imaging system (Phoenix Research Labs Inc.).

Statistics. Statistical analyses were performed using GraphPad Prism 6.0 (GraphPad Software), and individual data points were graphed using scatter plots as recommended by Weissgerber et al. (40). For the majority of data, a 1-way ANOVA with Tukey's or Dunnett's multiple comparisons test was used to assess statistical significance. A 2-tailed Student's $t$ test was used when the experiment involved only 2 groups, and the variation was similar in the 2 groups. A 2-tailed Welch's $t$ test was used when the variation in the 2 groups was dissimilar. A paired, 2-tailed $t$ test was used for the data shown in Figure 7, E-G. Welch's 1-way ANOVA and Games-Howell multiple comparisons test for unequal variances were used for the data shown in Figure 1, A and C, and Figure 7D. In Figure 2C, Figure 3A, Figure 4, $A$ and E, Figure 5D, and Supplemental Figure 2C, Supplemental Figure 3A, and Supplemental Figure 4, A and J, each data point in the graphs represents a single experiment in which the band intensity was normalized to its control, thereby producing a ratio between the 2 band intensities. We show the geometric mean and geometric 95\% CIs of these ratios ( $y$ axis is logarithmic). Statistical significance was assessed using the ratio paired, 2-tailed $t$ test, and the $P$ value is shown in each graph. Quantification for Figure 2, A and B, Figure 3D, and Figure 4, C and $\mathrm{D}$, is provided in the supplemental materials. A $P$ value of less than 0.05 was considered statistically significant.

Study approval. All animal experiments were conducted in accordance with the NIH Guide for the Care and Use of Laboratory Animals (National Academies Press, 2011), and all protocols were approved by the IACUCs of the University of Utah, the University of Wisconsin, and Absorption Systems.

\section{Author contributions}

WZ, DSS, JMW, MEH, SJO, and DYL were responsible for project conceptualization, experimental design, data analysis, and manuscript preparation. WZ, ZT, LKS, BER, AJ, JHY, YH, DMW, JRR, and $\mathrm{HZ}$ performed and collected data for in vitro experiments. DSS, JMW, WZ, JAB, TMM, and JL collected data for in vivo experiments. ZT and KRT made constructs. ALM, KO, ZT, and CD conducted the drug experiments. WZ, DYL, SJO, KO, LAL, and MEH obtained funding for the project.

\section{Acknowledgments}

We thank the Utah donors who generously gifted their eyes for the advancement of biomedical science. We thank D. Lim (University of Utah, Salt Lake City, UT) for preparation of the figures, comments, and consultation regarding responsible and effective display of the images. We thank P. Allaire, Thien Nhut $\mathrm{Vu}$, E. Jorgensen, W. Sundquist, and J. Kaplan (all from University of Utah, Salt Lake City, UT) for reading the manuscript. We thank C. Rodesch at the University of Utah Cell Imaging/ Fluorescence Facility for help with imaging. This work was funded by grants to DYL (R01CA163970, R01NS080893, U54HL112311, R01HL077671, ROLHL084516, R01AR064788, UL1TR000105, R43EY022516, R01CA202778, and R01HL130541), SJO (R01AR064788, R01CA202778, and R01HL130541) and WZ (R01HL077671 and R01EY025342) from the NIH, and grants to 
DYL from the Juvenile Diabetes Research Foundation (172011517), the American Asthma Foundation (09-0172), and the Burroughs Wellcome Fund (Clinical Scientist Award in Translational Medicine). KO received NIH grant U01NS083573 and R43EY022516. MEH received NIH grants R01EY015130 and R01EY017011. LAL received a VA Merit Award (1IOBX002151).
Address correspondence to: Weiquan Zhu, Building 533 Room 4130, 15 North 2030 East, Salt Lake City, Utah 84112, USA. Phone: 801.585.0869; Email: weiquan.zhu@u2m2.utah.edu. Or to: Shannon J. Odelberg, Building 533 Room 4130, 15 North 2030 East, Salt Lake City, Utah 84112, USA. Phone: 801.581.3598; Email: sodelber@genetics.utah.edu.
1. Inzucchi SE. Clinical practice. Diagnosis of diabetes. N Engl J Med. 2012;367(6):542-550.

2. Qaum T, et al. VEGF-initiated blood-retinal barrier breakdown in early diabetes. Invest Ophthalmol Vis Sci. 2001;42(10):2408-2413.

3. Bolinger MT, Antonetti DA. Moving past antiVEGF: novel therapies for treating diabetic retinopathy. Int J Mol Sci. 2016;17(9):E1498.

4. Lampugnani MG, Orsenigo F, Gagliani MC, Tacchetti C, Dejana E. Vascular endothelial cadherin controls VEGFR-2 internalization and signaling from intracellular compartments. JCell Biol. 2006;174(4):593-604.

5. Lee $\mathrm{S}$, et al. Autocrine VEGF signaling is required for vascular homeostasis. Cell. 2007;130(4):691-703.

6. Wheeler DB, Zoncu R, Root DE, Sabatini DM, Sawyers CL. Identification of an oncogenic RAB protein. Science. 2015;350(6257):211-217.

7. Bhattacharya $R$, et al. Regulatory role of dynamin-2 in VEGFR-2/KDR-mediated endothelial signaling. FASEB J. 2005;19(12):1692-1694.

8. Lanahan A, et al. The neuropilin 1 cytoplasmic domain is required for VEGF-A-dependent arteriogenesis. Dev Cell. 2013;25(2):156-168.

9. Ballmer-Hofer K, Andersson AE, Ratcliffe LE, Berger P. Neuropilin-1 promotes VEGFR-2 trafficking through Rab11 vesicles thereby specifying signal output. Blood. 2011;118(3):816-826.

10. Molendijk AJ, Ruperti B, Palme K. Small GTPases in vesicle trafficking. Curr Opin Plant Biol. 2004;7(6):694-700.

11. Schweitzer JK, Sedgwick AE, D'Souza-Schorey C. ARF6-mediated endocytic recycling impacts cell movement, cell division and lipid homeostasis. Semin Cell Dev Biol. 2011;22(1):39-47.

12. Donaldson JG, Jackson CL. ARF family G proteins and their regulators: roles in membrane transport, development and disease. Nat Rev Mol Cell Biol. 2011;12(6):362-375.

13. Guilluy C, Swaminathan V, Garcia-Mata R, O'Brien ET, Superfine R, Burridge K. The Rho GEFs LARG and GEF-H1 regulate the mechanical response to force on integrins. Nat Cell Biol. 2011;13(6):722-727.

14. Zhu W, et al. Interleukin receptor activates a MYD88-ARNO-ARF6 cascade to disrupt vascular stability. Nature. 2012;492(7428):252-255.

15. Davis CT, et al. ARF6 inhibition stabilizes the vasculature and enhances survival during endotoxic shock. J Immunol. 2014;192(12):6045-6052.

16. Yoo JH, et al. ARF6 is an actionable node that orchestrates oncogenic GNAQ signaling in uveal melanoma. Cancer Cell. 2016;29(6):889-904.

17. Lai AK, Lo AC. Animal models of diabetic retinopathy: summary and comparison. J Diabetes Res. 2013;2013:106594.

18. Hongu T, et al. Arf6 regulates tumour angiogenesis and growth through HGF-induced endothelial $\beta 1$ integrin recycling. Nat Commun. 2015;6:7925.

19. Ikeda $S$, et al. Novel role of ARF6 in vascular endothelial growth factor-induced signaling and angiogenesis. Circ Res. 2005;96(4):467-475.

20. Tahir SA, Park S, Thompson TC. Caveolin-1 regulates VEGF-stimulated angiogenic activities in prostate cancer and endothelial cells. Cancer Biol Ther. 2009;8(23):2286-2296.

21. Liao WX, Feng L, Zhang H, Zheng J, Moore TR, Chen DB. Compartmentalizing VEGF-induced ERK2/1 signaling in placental artery endothelial cell caveolae: a paradoxical role of caveolin-1 in placental angiogenesis in vitro. Mol Endocrinol. 2009;23(9):1428-1444.

22. Labrecque L, Royal I, Surprenant DS, Patterson C, Gingras D, Béliveau R. Regulation of vascular endothelial growth factor receptor-2 activity by caveolin-1 and plasma membrane cholesterol. Mol Biol Cell. 2003;14(1):334-347.

23. Grossmann AH, et al. The small GTPase ARF6 stimulates $\beta$-catenin transcriptional activity during WNT5A-mediated melanoma invasion and metastasis. Sci Signal. 2013;6(265):ra14.

24. Mannell HK, et al. ARNO regulates VEGFdependent tissue responses by stabilizing endothelial VEGFR-2 surface expression. Cardiovasc Res. 2012;93(1):111-119.

25. Béraud-Dufour S, et al. A glutamic finger in the guanine nucleotide exchange factor ARNO displaces $\mathrm{Mg} 2+$ and the $\beta$-phosphate to destabilize GDP on ARF1. EMBO J. 1998;17(13):3651-3659.

26. Mossessova E, Gulbis JM, Goldberg J. Structure of the guanine nucleotide exchange factor Sec7 domain of human arno and analysis of the interaction with ARF GTPase. Cell. 1998;92(3):415-423.

27. Hashimoto A, et al. GEP100-Arf6-AMAP1cortactin pathway frequently used in cancer invasion is activated by VEGFR 2 to promote angiogenesis. PLoS One. 2011;6(8):e23359.

28. Zhang X, Simons M. Receptor tyrosine kinases endocytosis in endothelium: biology and signaling. Arterioscler Thromb Vasc Biol. 2014;34(9):1831-1837.

29. Jo DH, Cho CS, Kim JH, Jun HO, Kim JH. Animal models of diabetic retinopathy: doors to investigate pathogenesis and potential therapeutics. J Biomed Sci. 2013;20:38.
30. Frevert CW, Wong VA, Goodman RB, Goodwin R, Martin TR. Rapid fluorescence-based measurement of neutrophil migration in vitro. JImmunol Methods. 1998;213(1):41-52.

31. Nemeth E, et al. Hepcidin regulates cellular iron efflux by binding to ferroportin and inducing its internalization. Science. 2004;306(5704):2090-2093.

32. van Weert AW, Geuze HJ, Groothuis B, Stoorvogel W. Primaquine interferes with membrane recycling from endosomes to the plasma membrane through a direct interaction with endosomes which does not involve neutralisation of endosomal $\mathrm{pH}$ nor osmotic swelling of endosomes. Eur J Cell Biol. 2000;79(6):394-399.

33. Roberts M, Barry S, Woods A, van der Sluijs P, Norman J. PDGF-regulated rab4-dependent recycling of alphavbeta 3 integrin from early endosomes is necessary for cell adhesion and spreading. Curr Biol. 2001;11(18):1392-1402.

34. London NR, et al. Targeting Robo4dependent Slit signaling to survive the cytokine storm in sepsis and influenza. Sci Transl Med. 2010;2(23):23ra19.

35. Scheppke L, et al. Retinal vascular permeability suppression by topical application of a novel VEGFR2/Src kinase inhibitor in mice and rabbits. J Clin Invest. 2008;118(6):2337-2346.

36. Maharjan S, et al. Sac-0601 prevents retinal vascular leakage in a mouse model of diabetic retinopathy. Eur JPharmacol. 2011;657(1-3):35-40.

37. Kim JH, Kim JH, Yu YS, Cho CS, Kim KW. Blockade of angiotensin II attenuates VEGFmediated blood-retinal barrier breakdown in diabetic retinopathy. JCereb Blood Flow Metab. 2009;29(3):621-628.

38. Tugal-Tutkun I, Herbort CP, Khairallah M, Angiography Scoring for Uveitis Working Group (ASUWOG). Scoring of dual fluorescein and ICG inflammatory angiographic signs for the grading of posterior segment inflammation (dual fluorescein and ICG angiographic scoring system for uveitis). Int Ophthalmol. 2010;30(5):539-552.

39. Hui F, et al. Quantitative spatial and temporal analysis of fluorescein angiography dynamics in the eye. PLoS One. 2014;9(11):e111330.

40. Weissgerber TL, Milic NM, Winham SJ, Garovic VD. Beyond bar and line graphs: time for a new data presentation paradigm. PLoS Biol. 2015;13(4):e1002128.

41. Sawamiphak S, et al. Ephrin-B2 regulates VEGFR2 function in developmental and tumour angiogenesis. Nature. 2010;465(7297):487-491. 